\title{
The Evolutionary Conserved Transmembrane BAX Inhibitor Motif (TMBIM) Containing Protein Family is Essential for the Development and Survival of Drosophila Melanogaster
}

\section{Li Zhang}

University Medical Center of the Johannes Gutenberg University Mainz

\section{Sebastian Buhr}

University Medical Center of the Johannes Gutenberg University Mainz

Vibha Prasad

Universitätsklinikum Aachen

Aaron Voigt

Universitätsklinikum Aachen

Axel Methner ( $\sim$ axel.methner@gmail.com )

University Medical Center of the Johannes Gutenberg University Mainz

\section{Research Article}

Keywords: RECS1, PP1201, LFG3, MST100, MSTP100, NMP35, LFG, FAIM2, LFG2, NGP35, KIAA0950, LFG1, HNRGW, NMDARA1, MGC99687, GRINA, GBP, GAAP, S1R, LFG4, ZPRO, CGI-119, GHITM, MICS1, DERP2, My021, PTD010, HSPC282, FLJ26584, DKFZp566C0746

Posted Date: December 3rd, 2020

DOI: https://doi.org/10.21203/rs.3.rs-113374/v1

License: (c) (i) This work is licensed under a Creative Commons Attribution 4.0 International License. Read Full License

Version of Record: A version of this preprint was published at Frontiers in Cell and Developmental Biology on September 3rd, 2021. See the published version at https://doi.org/10.3389/fcell.2021.666484. 


\section{The evolutionary conserved transmembrane BAX inhibitor motif (TMBIM) containing protein family is essential for the development and survival of Drosophila Melanogaster}

\footnotetext{
Li Zhang ${ }^{1,2}$, Sebastian Buhr ${ }^{1,2}$, Vibha Prasad ${ }^{3}$, Aaron Voigt ${ }^{3,4^{*}}$, and Axel Methner ${ }^{1^{*} \#}$ ${ }^{1} J o h a n n e s$ Gutenberg University Medical Center Mainz, Institute for Molecular Medicine, Langenbeckstr. 1, D-55131 Mainz, Germany.

${ }^{2}$ Institute of Developmental Biology and Neurobiology (iDN), Johannes Gutenberg University Mainz, Biozentrum 1, Hanns-Dieter-Hüsch-Weg 15, D-55128 Mainz, Germany

${ }^{3}$ Department of Neurology, RWTH University Aachen, Pauwelsstr. 30, D-52074 Aachen, Germany.

${ }^{4}$ JARA-Institute Molecular Neuroscience and Neuroimaging, Forschungszentrum Jülich $\mathrm{GmbH}$ and RWTH Aachen University, Pauwelsstraße 30, 52074 Aachen, Germany.

Running title: Characterization of the TMBIM family in Drosophila melanogaster

\# Correspondence should be addressed to: Axel Methner MD, Johannes Gutenberg University Medical Center Mainz, Institute for Molecular Medicine, Langenbeckstraße 1, D-55131 Mainz, Germany, Tel.: +49-6131-17-2695, Fax: +49-6131-17-5967, Email: axel.methner@gmail.com

* Equally contributed
} 


\section{Abstract}

The Transmembrane BAX Inhibitor Motif (TMBIM) protein family consists of six evolutionarily conserved hydrophobic proteins that affect programmed cell death and the regulation of intracellular calcium levels. The bacterial orthologue BsYetJ is a $\mathrm{pH}$-dependent calcium channel. We here identified six TMBIM family members in Drosophila melanogaster and studied the effect of their RNAi-mediated knockdown using ubiquitous and tissue-specific drivers. Mammalian TMBIM6 and TMBIM5 have obvious orthologs while this is more dubious for the other family members. Ubiquitous knockdown of family members dmTMBIM1,4,5, and 6 caused failed eclosing and tissue-specific knockdown resulted in a dramatically decreased lifespan. On the contrary, knockdown of dmTMBIM3, surprisingly, extended lifespan. Only knockdown of dmTMBIM6 increased the ER calcium levels of Pdf neurons. Neural knockdown of dmTMBIM2,3, and 4 increased ER stress, as indicated by increased Xbp1 splicing. Interestingly, TMBIM1 and TMBIM6 have a very similar expression pattern and their knockdown phenocopied each other. Also, knockdown of TMBIM1 resulted in upregulation of TMBIM6 and vice versa further suggesting a genetic interaction between these two genes. Our data demonstrate that most TMBIM proteins are essential for fly development and survival but, despite their shared protein structure, affect cell survival through different mechanisms.

Key words: RECS1; PP1201; LFG3; MST100; MSTP100; NMP35; LFG; FAIM2; LFG2; NGP35; KIAA0950; LFG1; HNRGW; NMDARA1; MGC99687; GRINA; GBP; GAAP; S1R; LFG4; ZPRO; CGI-119; GHITM; MICS1; DERP2; My021; PTD010; HSPC282; FLJ26584; DKFZp566C0746 


\section{Background}

Transmembrane BAX Inhibitor Motif (TMBIM) proteins are found in all of nature's kingdoms, in bacteria, plants and animals ${ }^{1}$. They are characterized by the presence of multiple transmembrane spanning domains, share a specific signature (prosite PDOC00957) between the third and fourth transmembrane domain, and possess a semi-hydrophobic putative loop domain at their $\mathrm{C}$-terminus. TMBIM proteins are localized to distinct intracellular membranes: TMBIM1 at the Golgi apparatus ${ }^{2}$ and lysosome ${ }^{3,4}$, TMBIM2 and 3 at the Golgi apparatus ${ }^{2}$,

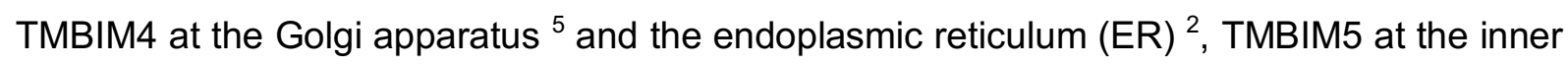
mitochondrial membrane ${ }^{2,6}$, and TMBIM6 at the $\mathrm{ER}^{2,7,8}$.

The name-giving human protein Bax inhibitor-1 (BI-1, TMBIM6) was originally identified in a yeast-based screen for its ability to inhibit cell death caused by overexpression of the proapoptotic protein Bax, hence its name ${ }^{7}$. TMBIM6 affects the $\mathrm{ER} \mathrm{Ca}^{2+}$ content; its knockout increases the $\mathrm{ER} \mathrm{Ca}^{2+}$ content ${ }^{9}$ while its over-expression reduces it ${ }^{10}$. This function is mediated by its C-terminal loop domain ${ }^{8}$ and altered by $\mathrm{pH}^{11,12}$. Recent structural and biochemical work with a bacterial TMBIM protein from Bacillus subtilis, BsYetJ, also confirmed a function as a $\mathrm{pH}$-dependent ion channel. Crystal structures obtained in closed and open conformations were reversibly interconvertible by change of $\mathrm{pH}$ and recombinant BsYetJ reconstituted in proteoliposomes mediated $\mathrm{Ca}^{2+}$ influx in a $\mathrm{pH}$-dependent manner ${ }^{13}$. Also TMBIM3, first described as glutamate receptor ionotropic NMDA protein 1 (GRINA) ${ }^{14}$, and TMBIM4 (better known as GAAP ${ }^{5}$ ) have been reported to modulate the resting ER $\left[\mathrm{Ca}^{2+}\right]$ and the release of $E R \mathrm{Ca}^{2+15,16}$.

Other members of the TMBIM protein family in mammals are TMBIM1/RECS1 (responsive to centrifugal force and shear stress gene 1 protein) ${ }^{17}$, TMBIM2/LFG (life guard) ${ }^{18}$, and TMBIM5/GHITM (growth hormone-inducible transmembrane protein) also known as MICS1 (for mitochondrial morphology and cristae structure 1$)^{6}$. All family members play a role in the regulation of programmed cell death, specifically upon ER stress. TMBIM1 apparently interacts with the Fas ligand receptor CD95/Apo1 in the Golgi apparatus thus preventing its transport to the cell membrane ${ }^{17}$. Knockdown of TMBIM2 induces more active caspase $8{ }^{19}$. Overexpression of TMBIM3 restrains apoptosis specifically induced by ER stress ${ }^{15}$. TMBIM4 over-expression prevents apoptosis induced by different kinds of stimuli - the proapoptotic Bax, staurosporine, doxorubicin, C2-ceramide, as well as TNF $\alpha$ and Fas ligand while its knockdown results in spontaneous apoptosis ${ }^{5}$. TMBIM5, a family member targeted to the mitochondrial inner membrane protein, maintains mitochondrial morphology and decreases the release of cytochrome $c$ from mitochondria which indirectly links it to the induction of apoptosis ${ }^{6}$ possibly by interfering with the mitochondrial protein synthesis machinery ${ }^{20}$. Overexpression of TMBIM6 inhibits cell death caused by BAX, etoposide, staurosporine and 
growth factor withdrawal, possibly by forming a complex with the anti-apoptotic proteins BCL2 and BCL-X ${ }^{7}$. Knockout of TMBIM6 increases the susceptibility for ER stress ${ }^{9}$ which was later attributed to its role as a direct inhibitor of the ER stress sensing protein inositol-requiring protein $1 \alpha(\operatorname{IRE} 1 \alpha)^{21}$, a serine/threonine protein kinase and endoribonuclease inserted in the ER membrane that represents the most ancient and conserved branch of the unfolded protein response (UPR).

TMBIM1 knockout (KO) mice are prone to cystic medial degeneration ${ }^{3}$ and accelerated metabolic cardiomyopathy caused by high fat diet-induced through activating proinflammatory factor cytokines such as the nuclear factor-KB (NF-KB) ${ }^{22}$. Cardiac-specific TMBIM1 KO causes a pathological cardiac hypertrophy by lack of lysosomal degradation of the activated proinflammatory and prohypertrophy factor Toll-like receptor 4 (TRL4) ${ }^{23}$, while TMBIM1 overexpression in hepatocytes of mice inhibited high-fat diet-induced insulin resistance, hepatic steatosis and inflammation by promoting lysosomal degradation of TRL4 ${ }^{4}$. TMBIM2 $\mathrm{KO}$ mice are viable but suffer from cerebellar atrophy caused by a reduced internal granular layer thickness and arrested Purkinje cell development correlating with increased levels of activated caspases 8 and 3 in the affected cells ${ }^{19}$. Furthermore, cerebellar slices from TMBIM2 KO mice are more susceptible to Fas-mediated cell death ${ }^{19}$ and more vulnerable to 1-methyl4-phenyl-1,2,3,6-tetrahydropyridine (MPTP), an animal model of Parkinson's disease ${ }^{24}$. TMBIM3 knockdown in the zebra fish results in embryonal lethality caused by disordered apoptosis ${ }^{15}$. Using the Drosophila melanogaster model system, KD of the dmTMBIM3 or dmTMBIM6 alone resulted in a reduced hatching rate only when exposed to tunicamycin whereas dmTMBIM3 and 6 double KD flies displayed a decreased hatching rate per se which was even more pronounced upon treatment with tunicamycin ${ }^{15}$. We have shown previously that TMBIM6 KO mice are obese and suffer from leukopenia and erythrocytosis, showing more splenic marginal zone $\mathrm{B}$ cells and nuclear translocation of NF-kB, changes that correlated with increased cytosolic and ER Ca ${ }^{2+}$ levels but not with constitutive ER stress ${ }^{25,26}$

So far, no conclusive, comparative analysis of the role of the protein family in $\mathrm{Ca}^{2+}$ homeostasis and apoptosis has been conducted on an organismal level. For this study, we identified six TMBIM family members in the fruit fly Drosophila melanogaster and studied the effect of ubiquitous and tissue-specific RNAi-mediated knockdown on development and lifespan as well as on ER $\mathrm{Ca}^{2+}$ and ER stress levels. Our data demonstrate that most TMBIM proteins are essential for fly development and survival but affect survival through different mechanisms despite their shared protein structure. 


\section{Results}

Identification of all TMBIM proteins in the Drosophila melanogaster genome

Using the BLASTP algorithm (www.blast.ncbi.nIm.nih.gov) with human TMBIM1-6 as input, we identified seven putative fly TMBIM proteins in the Drosophila melanogaster genome and constructed a phylogenetic tree of all fly and human TMBIM proteins using the bacterial homologue BsYetJ as outlier (Figure 1). From this analysis it appears that the fly TMBIM proteins fall into two distinct groups consisting of TMBIM1-3 and TMBIM4-6 similar to the vertebrate TMBIM proteins ${ }^{2}$. The fly proteins CG2076 and CG1287 are likely orthologues of human TMBIM5 and CG7188 appears to be the orthologue of human TMBIM6. CG30379 most closely represents the orthologue of human TMBIM4 although these two proteins are much less related than TMBIM5 and 6 with their respective orthologues. The relationships between fly CG3798, CG3814, CG9722 and human TMBIM1-3 are more dubious as all fly proteins and all human proteins are more closely related to each other than to their putative orthologues. In an effort to match fly and human orthologues, we therefore added serial BLASTP of two sequences to the analysis which suggested that human TMBIM2 corresponds most likely to CG3814 and human TMBIM3 to CG3798 leaving CG9722 as the most probable human TMBIM1 orthologue. This grouping is also supported by the relative relationships between the proteins in their respective branches where human TMBIM1 is more closely related to human TMBIM2 similar to fly CG9722 being more closely related to fly CG3814. We conclude that a definite attribution of the fly proteins to their human counterparts is obvious for dmTMBIM5 and 6 and probable for dmTMBIM4. It is however not useful for the branch consisting of CG3798, CG3814, and CG9722 (TMBIM1-3), as these proteins are much more closely related to each other than to the human proteins and probably evolved from a common ancestor.

\section{Expression pattern and knockdown phenotype of fly TMBIM proteins}

To systematically clarify the function of the fly TMBIM proteins in a comparative manner, we studied their temporal and tissue-specific expression by exploiting the extensive wealth of information provided by FlyAtlas (www.flyatlas.org). We also determined alterations in developmental viability, lifespan, locomotion, circadian rhythm, in response to ubiquitous or tissue-specific knockdown (KD) achieved by the UAS-Gal4 system of tissue-specific drivers ${ }^{27}$ combined with two specific RNAi lines for each gene wherever possible. Ubiquitous KD was accomplished by using tub-Gal4, somatic muscular KD with Mef2-Gal4 (also including pacemaker LNv and Pdf neurons), pan-neural KD with elav-Gal4, and KD in neurons that control circadian activity with Pdf-Gal4 flies. Successful KD was controlled by quantitative PCR.

CG7188 (dmTMBIM6) is expressed in all tissues and at all developmental stages. In adult flies, it is most strongly expressed in the testes and the accessory glands (Figure 2a). KD by two 
independent RNAi lines showed the same phenotypes: ubiquitous KD resulted in no larvae, muscular KD caused uneclosed pupa. The efficiency of KD was shown by quantitative RTPCR (qPCR) on whole larvae of muscular KD (Figure 2b). In both RNAi lines, pan-neural KD of CG7188 caused a lower eclosing rate and most surviving flies presented with an uninflated wing phenotype and lived shorter (Figure 2c). Crawling speed of larvae with muscular KD was similar to controls (Figure 2d). KD of CG7188 in Pdf neurons resulted in an overall lower activity, especially in the dark phase, resulting in a less pronounced circadian rhythm (Figure $2 \mathrm{e})$.

CG2076 (dmTMBIM5) is also expressed ubiquitously at all developmental stages. In adult flies, the highest expression levels are found in the carcass and the testes (Figure 3a). With CG2076, only ubiquitous KD caused lethality (Figure 3b). We therefore isolated RNA from flies with muscle-specific KD and demonstrated efficient KD in two independent RNAi lines (Figure 3b). Neural KD in both RNAi lines caused lower eclosing rate of pupa and shortened lifespan (Figure 3c). Muscle-specific KD reduced the crawling speed of larvae and climbing speed of young (2-day-old) and old (30-day-old) adults, and also significantly reduced the lifespan (Figure 3d). KD of CG2076 in Pdf neurons also caused an overall lower activity, and a less pronounced circadian rhythm (Figure 3e).

CG30379 (putative dmTMBIM4) is expressed only in very low amounts in the central nervous system (CNS) from late L3 larva to the early pupa stage according to the database (Figure 4a). Here, we again used two different RNAi lines and while ubiquitous KD by RNAi \#1 led to lethality, RNAi \#2 did not (Figure 4b). Also, tissue-specific KD did not cause lethality (Figure $4 b)$. Using qPCR on mRNA obtained from adult heads of neural KD by RNAi\#1 demonstrated a $50 \%$ reduction and by RNAi\#2 a $60 \%$ reduction (Figure 4b). Neural KD of CG30379 by RNAi\#1 resulted in a shortened lifespan, as well as a tendency of lower eclosing rate of pupa; KD by RNAi\#2 had no effect despite being rather more efficacious (Figure 4c). Mef2 expresses not only in muscles, but also in adult pacemaker neurons ${ }^{28}$. Mef2-driven RNAi\#1 reduced lifespan in adult flies, but did not affect the crawling speed of larvae and climbing speed of adults (Figure 4d). KD of CG30379 in Pdf neurons abolished the circadian rhythm (Figure 4e). CG3798 (putative dmTMBIM3) is ubiquitously and in adults most strongly expressed in heads (Figure $5 a$ ) and we observed no lethality with ubiquitous or tissue-specific drivers (Figure 5b) despite a strong $90 \%$ reduction by RNAi\#1 and a mild $60 \%$ reduction by RNAi\#2 in mRNA levels in adult flies with ubiquitous KD (Figure 5b). Neural KD had no effect on the eclosing rate for both lines, and interestingly prolonged lifespan although KD by RNAi\#2 had a more ambiguous effect (Figure 5c). Muscle-specific KD had no effect on the crawling speed of larvae or the climbing ability and longevity for the adults (Figure 5d). However, KD of CG3798 in Pdf neurons increased the activity of the flies in general, especially during the dark phase (Figure $5 e)$. 
CG3814 (putative dmTMBIM2) is expressed mainly in the L3 wandering larvae and adult digestive system (Figure 6a). Ubiquitous or tissue-specific KD caused no lethality (Figure 6b). Quantitative PCR from whole adult flies with ubiquitous KD revealed an almost complete knockdown with RNAi line \#1 and approximately 50\% with RNAi line \#2 (Figure 6b). Neural KD had no effect on the eclosing rate but strongly reduced lifespan in the \#1 RNAi line and mildly decreased the lifespan in the \#2 RNAi line, which is in line with the KD efficiency of the according RNAi line (Figure 6c). Muscle-specific KD of CG3814 slightly reduced lifespan for adult flies but had no effect on crawling speed of larvae and climbing speed of adults (Figure $6 \mathrm{~d})$. Pdf-neural KD of CG3814 also did not affect the circadian rhythm and activity of the flies (Figure 6e).

CG9722 (putative dmTMBIM1) is scarcely detectable before the L3 larva stage but starts to increase thereafter only in adult males where it is predominantly expressed in the testes (Figure 7a). Very similar to CG7188 (dmTMBIM6) ubiquitous KD and muscular KD of CG9722 led to developmental lethality. RNA was isolated from whole L3 larvae of RNAi\#1(v28361) muscle-specific KD, showing 95\% reduction (Figure 7b). Quantitative PCR using RNAi\#2 (BL6679) larvae however demonstrated even higher levels of CG9722 mRNA (Figure S1a). We therefore obtained CG9722 [MI08483] (BL44979), a mimic line, with a mimic cassette insertion in the exon region of CG9722, which also exhibited no effect on the mRNA levels of CG9722 in homozygous flies (Figure S1b). Using PCR on genomic DNA, we found that the mimic cassette is present in the genome but apparently not at the CG9722 genomic locus (Figure S1c). Therefore, we only studied the effect of RNAi\#1, neural KD resulted in a normal eclosing rate of pupa, but a reduced lifespan. Interestingly, the same uninflated wing phenotype observed in neural KD of CG7188 flies was also present in neural CG9722 KD flies (Figure 7c). Larvae of muscular KD had no problem with crawling, but the very few survivors $(n=12)$ showed also a shorter lifespan (Figure 7d). KD of CG9722 in Pdf neurons resulted in an overall lower activity especially in the dark phase, and a less pronounced circadian rhythm (Figure 7e). Knockdown of CG9722 phenocopied CG7188 KD suggesting that the two genes might cooperate with each other. We therefore measured the transcriptional level of one gene when the other gene was knocked down, and found out that transcriptional level of CG9722 increased in CG7188 muscular KD L3 larvae and vice versa (Figure 7f,g) further suggesting a genetic interaction between the two genes.

\section{Effects of TMBIM knockdown on ER calcium levels and ER stress}

To elucidate how KD of the TMBIM proteins disrupts cellular function, we studied the two most unequivocal functions described for these proteins in other organisms, alterations of the ER $\mathrm{Ca}^{2+}$ levels and induction of ER stress. $\mathrm{ER} \mathrm{Ca}^{2+}$ levels were quantitated by targeting the genetically-encoded ER Ca ${ }^{2+}$ sensor erGAP3 ${ }^{29}$ to Pdf neurons in TMBIM-specific RNAi lines (Figure 8a). Pdf neurons are easily detected and fluorescence can be quantified by confocal 
microscopy ${ }^{30,31}$. As we observed changes in the circadian rhythm upon pdf-neuronal KD in dmTMBIM3-6 flies, we expected alterations in at least these four fly lines. We examined $\mathrm{Ca}^{2+}$ levels in the same age range used to monitor changes in circadian rhythm to render comparisons feasible. This revealed an increase in ER Ca ${ }^{2+}$ levels only in CG7188 (dmTMBIM6) KD flies (Figure 8b). ER stress was assessed by using qPCR assays for spliced and unspliced Xbp1 (CG9415) ${ }^{32}$ (Figure 8c) with mRNA from the heads of neuronal (elav-gal4) KD 2-day-old flies of each TMBIM family member. This demonstrated more spliced Xbp1 indicating a higher activity of IRE ${ }^{33}$ and thus increased ER stress levels after neural KD of CG30379 (putative dmTMBIM4), CG3814 (putative dmTMBIM2) and CG3798 (putative dmTMBIM3) (Figure 8d).

\section{Discussion}

This study is the first conclusive, comparative analysis of the role of the TMBIM protein family in vivo and it brought forth a number of interesting facts that shed further light on this interesting but due to its very hydrophobic nature difficult-to-study protein family. Surprising results were that knockdown of CG9722 (putative dmTMBIM1) and CG7188 (dmTMBIM6) results in almost the same phenotype (summarized in Figure 8e) suggesting a shared pathway, and that knockdown of CG3798 (putative dmTMBIM3) resulted in an increased and not a decreased lifespan as observed for most other family members.

To rule out off-target effect, we used two independent RNAi lines for each TMBIM gene obtained from international Drosophila resource centers. In GD lines, short hairpin RNAs (shRNAs) are driven by a $10 x$ UAS promoter and randomly inserted into $X, 2^{\text {nd }}$, or $3^{\text {rd }}$ chromosome of the flies ${ }^{34}$. In Trip lines, the shRNAs are also driven by 10x UAS but inserted at attP2 (68A4) or attP40 (25C6) sites in the fly genome ${ }^{35}$. The Trip Valium20 shRNA vector contains a modified scaffold of the microRNA miR-1 resulting in a stronger knockdown efficiency than the Valium 10 vector ${ }^{35}$. For CG7188/dmTMBIM6, two GD RNAi lines expressing the same shRNA (dna1660) but randomly inserted into the $X, 2^{\text {nd }}$, or $3^{\text {rd }}$ chromosomes (Table S1) had the same efficiency and resulted in identical phenotypes suggesting it was not due to an off-target effect but the knockdown of dmTMBIM6. For CG2076/dmTMBIM5, one GD RNAi line and one Trip RNAi line also showed the same knockdown efficiency and phenotypes. For CG30379/dmTMBIM4, two Trip lines with different promoter strengths were used; RNAi\#1 driven by valium 20 and RNAi\#2 driven by valium 10 . Valium 20 results in a stronger knockdown than Valium10 in the soma and the germline ${ }^{35}$ and in line with this, ubiquitous knockdown of dmTMBIM4 by tub>>RNAi\#1 indeed resulted in lethality, whereas tub>>RNAi\#2 (valium10) flies were still viable although the knockdown efficiency quantitated by qPCR of mRNA obtained from head samples from elav-Gal4 driven flies was similar. We chose head samples from elav-Gal4 driven flies for both RNAi lines to make the quantification comparable. A 
possible explanation could be the lower eclosing rate of elav>> RNAi\#1 flies where flies with the most dramatic phenotypes could not be included in the RNA samples because they died at eclosing. We are therefore confident that the observed phenotype is valid. For CG3798/dmTMBIM3 and CG3814/dmTMBIM2, one GD and one Trip line were used. In both cases, knockdown efficiency positively correlated with the phenotype. For CG9722/dmTMBIM1 as well one GD and one Trip line were used. Here, knockdown by RNAi\#1 was very efficient and resulted in a strong phenotype which resembled dmTMBIM6 knockdown flies but RNAi\#2 did not result in a knockdown (Figure S1a) or phenotype (data not shown). We therefore ordered a fly line with a MiMIC cassette inserted in the single exon of CG9722, hoping that it would result in a complete knockout. Unfortunately, we found out that this line contains a MiMIC cassette somewhere in the genome but not at the CG9722 locus (Figure S1c). There was also no knockdown of CG9722 in these flies (Figure S1b) nor any detectable phenotype. Therefore, we studied only RNAi\#1 for CG9722. Because of the shared phenotypes between TMBIM1 KD and TMBIM6 KD and the strong genetic interaction, we consider an off-target effect to be unlikely but this deserves further attention e.g. by generating a CRISPR/Cas9 knockout.

CG7188 (dmTMBIM6) is an ER membrane protein expressed in all developmental stages and in all tissues of Drosophila melanogaster. Accordingly, its ubiquitous but also muscle-specific $\mathrm{KD}$ was not reconcilable with life. This is however in contrast to previous work where ubiquitous KD using RNAi v3235 driven by the tub promoter, thus exactly the same setup as used in our study, had no effect despite an mRNA reduction of $80 \%$ in whole larvae ${ }^{15}$. We used v3235 and v37108, which use the same shRNA (dna1660, 320bp) inserted randomly., and found that ubiquitous KD by both RNAi lines driven by tub-Gal4 caused lethality. We therefore used fly heads of neural KD by elav-Gal4 to quantify mRNA, which demonstrated a reduction of CG7188 by around 70\% for both lines. In both RNAi lines, neuron-specific KD shortened lifespan and resulted in a strong wing phenotype. These results suggest that TMBIM6, at least in the fly, has important housekeeping functions which cannot be covered by other TMBIM proteins. In mice, however, ubiquitous knockout is compatible with life ${ }^{9,25}$. As mentioned above, an interesting result of our study was the almost complete phenocopy of dmTMBIM6 and dmTMBIM1 knockdown with very similar patterns of lethality, neurodegeneration and the same peculiar wing phenotype. Both proteins have a similar expression pattern and are most prominently expressed in testes and in the imaginal larval disc with dmTMBIM1 being consistently expressed at much lower levels than dmTMBIM6. Both proteins did not cause constitutive Xbp1 splicing and while dmTMBIM6 KD exhibited increased ER $\mathrm{Ca}^{2+}$ levels there was only a tendency towards such increased ER $\mathrm{Ca}^{2+}$ levels in dmTMBIM1 KD. On the other hand, the $\mathrm{Ca}^{2+}$ experiments were conducted in pdf neurons, which was actually the only $\mathrm{KD}$ phenotype where dmTMBIM6 and dmTMBIM1 KD were significantly different. Whereas 
dmTMBIM6 KD in pdf neurons resulted in an overall reduced activity, no such phenotype was evident in dmTMBIM1 KD. In our view, it appears possible that the two proteins depend on each other which is also in line with the qPCR data where we observed a compensatory upregulation of the respective counterpart. In mice as well, TMBIM1 (also known as RECS1) and TMBIM6 have similar expression patterns and cause similar phenotypes when knocked out. Mouse TMBIM1 has a very ubiquitous expression pattern ${ }^{3}$ similar to $\mathrm{BI}-1$, the proinflammatory cytokine NF-KB is increased in both TMBIM1 ${ }^{22}$ and TMBIM6 KO mice ${ }^{25}$. This hypothetical cooperation is nonetheless difficult to fathom as both proteins appear to be localized to different subcellular compartments, TMBIM6 to the ER and TMBIM1 to the Golgi apparatus ${ }^{2}$ although it must be stated here that these results were obtained using forced overexpression of tagged proteins in immortalized cell culture which might of course alter the subcellular localization. TMBIM1 has even been found the lysosome/endosome compartment shown by co-localization of GFP-tagged hTMBIM1 and lysotracker-Red in HT1080 cells ${ }^{3}$ and mCherry-tagged TMBIM1 and lysosomal protein LAMP2 in H9C2 ${ }^{4}$ and in Huh7 cells ${ }^{23}$. There are unfortunately no working antibodies against TMBIM6, it is therefore possible that endogenous TMBIM6 and TMBIM1 might be found in other compartments besides the ER and in the Golgi apparatus, e.g. the lysosome. Regarding the role of TMBIM6 in ER stress and $\mathrm{Ca}^{2+}$ homeostasis, our results are in line with previous findings obtained in a $\mathrm{Bl}-1$ knockout mouse where we found increased ER $\mathrm{Ca}^{2+}$ levels in phenotypically different cells, lymphocytes, but no hint of constitutive ER Stress ${ }^{25}$. This does of course not rule out an increased susceptibility of TMBIM6-deficient cells to ER stress as shown previously in mice ${ }^{9}$ and in flies, where a reduced hatching rate was observed in CG7188 KD flies subjected to additional ER stress by feeding flies tunicamycin ${ }^{15}$.

CG303079 (putative dmTMBIM4) is also an ER and Golgi membrane protein similar to TMBIM6 which is, at least according to the expression data obtained from Flybase, only expressed in the pupal nervous system and this even at very low levels. Nevertheless, ubiquitous KD caused lethality and neural KD decreased the lifespan suggesting an important role despite its scarcity. Global dysregulation (up and down) of hGAAP mRNA are detected in various kind of cancers according to www.oncomine.com. Although the human counterpart, hGAAP, has a very similar topology ${ }^{36}$ and similar effects on the cellular $\mathrm{Ca}^{2+}$ homeostasis than TMBIM6/BI-1 ${ }^{16}$, we observed no effect on the ER Ca ${ }^{2+}$ content but increased ER stress, suggesting that TMBIM4 regulates ER stress not though ER $\mathrm{Ca}^{2+}$.

Finally, CG3798 (putative dmTMBIM3) also deserves some discussion. In our hands, ubiquitous KD of CG3798 did not cause lethality and did not affect the hatching rate. Also in a previous study using the same RNAi line, a mild reduction of hatching rate was only revealed when the larvae were fed tunicamycin, an N-glycosylation inhibitor that induces ER stress ${ }^{15}$ in line with our finding that Xbp1 splicing was enhanced in neural KD flies. Interestingly, we also 
found that KD of CG3798 in Pdf-neurons induced hyper-activity of flies without changing ER $\mathrm{Ca}^{2+}$, and neural KD of CG3798 even extended the lifespan of flies which is in line with very recent findings that reported that increased spliced Xbp1 in a specific subset of glial cells extended the longevity of $C$. elegans ${ }^{37}$. Last but not least, prolonged lifespan and robust activities of knocking down CG3797 (probable dmTMBIM3) in the flies definitely shed light on the research of aging, and further studies should be conducted on the mechanism that why increased Xbp1 splicing in this case is protective for the living.

In summary, our study proves the importance of the TMBIM family members in many different organs and tissues of the fly. The fact that the fly possesses essentially the same set of TMBIM proteins as mammals advocate that these proteins have similar functions and imply that some of our findings might also be of importance for humans.

\section{Methods}

\section{Phylogenetic analysis}

Multiple sequence alignment focusing on amino acid conservation was performed using the online tool PRALINE using the Blosum62 scoring matrix with default settings ${ }^{38}$. Alignments were preprocessed using PSI-BLAST with 3 iterations and an E-value cutoff of $0.01{ }^{39}$. The phylogenetic tree was constructed using the online tool phylogeny.fr ${ }^{40}$. In short, the tool aligns the sequences using MUSCLE (3.7) with default settings ${ }^{41}$. After alignment, ambiguous regions were removed by Gblocks (v0.91b) and reconstructed in a phylogenetic tree using the maximum likelihood method in PhyML (v3.0 aLRT). Graphics were created using TreeDyn (v198.3). The multiple sequence alignment of TMBIMs across species was created using MAFFT version 6 using the E-INS-i setting and the alignment trimmed with JalView 2.5 at a cut-off value of $85 \%$ gaps. For the analysis of the secondary structure of the TMBIM family, the amino acid sequence of each TMBIM protein family member was entered as plain text into the 'Prediction of Transmembrane Regions and Orientation' (TMpred) application (ExPASy) and the prediction graphic of the preferred model with the highest score was chosen.

\section{Fly strains and behavior}

Fly stocks were obtained from the Bloomington Stock Center (BL) and the Vienna Drosophila Resource Centre (v): tub-GAL4 (BL5138), elav-GAL4 (BL458), Mef2-GAL4 (donated by Olaf Vef), Pdf-GAL4 (BL6899), UAS-CG7188-RNAi (v3235, v37108), UASCG2076-RNAi (v50221, BL64564), UAS-CG30379-RNAi (BL36679, BL28988), UAS-CG3798RNAi (v28365, BL28361), UAS-CG3814-RNAi (v4671, BL33354), UAS-CG9722-RNAi (BL6679 and BL28052), CG9722[MI08483] (BL44979), UAS-always early-RNAi (v13673), UAS-erGAP3 (donated by M. Teresa Alonso). Aging: the flies for aging assay were transferred to fresh food and the number of dead flies was recorded every three days. $n$ is indicated in the 
figures. Eclosing: pupa eclosing rate was observed until the $(n-1) \times 2$ day ( $n$ is the day of first F1 hatching) after crossing. Wing phenotype was scored at day 2 . Each group/cross has 50200 flies, and at least 3 groups/crosses per genotype were counted. Crawling: the speed of larva crawling was determined by the length of the route within 1 minute after centering the larva. Each dot in the graph represents one larva. Climbing: the speed of adult climbing was analyzed by video recording of flies in a $15 \mathrm{ml}$ Falcon tube after being tapped down on the bench. Each tube contained 10 flies and the video was recorded for $15 \mathrm{~s}$. Each dot in the graph indicated the average of speed of the 10 flies in one tube. 5 groups per genotype per age were examined. All behavior assays were conducted at the same time of the day, gender was randomly unbiased mixed, and the control groups were always included.

\section{Circadian clock}

A single 2-day-old male adult fly was placed in a tube with a diameter of $5 \mathrm{~mm}$ sealed by agar food on one side and a cotton lid on the other. The tube was placed in a Drosophila Activity monitor (DAM2, TriKinetics Inc USA) in an incubator with light(6-18 o'clock)/dark(18 o'clock to next day 6 o'clock) cycles. Locomotor events were recorded by an infrared beam placed in the middle of each tube every 30 min for 10 days. 15 flies per genotype.

\section{Microscopy}

Brains of 2-day-old Pdf $>>$ UAS-erGAP3,UAS-RNAi adult fly were dissected as described in ${ }^{31}$ and imaged by Leica SP2 with laser intensity $20 \%$, Alex488, Gain $20 \%$. Confocal images were quantified by NIH ImageJ. Mean fluorescence intensity of erGAP3 of the Pdf neuron cluster from each animal was plotted by GraphPad Prism.

\section{Semi-quantitative RT-PCR}

Total RNA was extracted using the ZR RNA MiniPrep kit (ZYMO RESEARCH) and cDNA synthesized from $10 \mathrm{ng} / \mu \mathrm{l}$ RNA using the High Capacity cDNA Reverse Transcription Kits (Life Tech). qPCR was conducted by Takyon No Rox Probe Master Mix dTTP (Eurogentee) with 2 $\mu \mathrm{l}$ of cDNA and final concentration of $570 \mathrm{nM}$ Primer and $100 \mathrm{nM}$ Probe. The primers were from Eurofin and probes from the Universal Probe Library (Roche). The primers and probe pairs are as following (gene, probe ID, sequence of forward primer, sequence of reverse primer): CG7188, \#36, gctgattatactgacgcaaaagc, ggataatctgattgagctatttgg; CG2076, \#57, aaccagcatcgcggataat, aaaaggcataggtgctctgg; CG30379, \#76, tttggcccaattactgttca, cttggtgggttggtggtaag; CG3814, \#40, cggccacctgttcagtagtt, gtgtatgcgccagtttcatc; CG3798, \#131, caccgagctgctgtgtatgt, cgaaaccgctgtggagtaa; CG9722, \#87, tgataaacgggtgcgtctg, agtatggccaccacaaatatcc; housekeeping gene, Rp49 (CG7939), \#117, cggatcgatatgctaagctgt, gcgcttgttcgatccgta; spliced Xbp1, \#116, gatctgccgcagggtatacaac, ggccacaactttccagagtg; nonspliced Xbp1, \#89, ctgtgcgtccaccaacct, tgtgtccacctgttgtataccc. 


\section{Statistical Analysis}

For two group comparison, unpaired t-test was performed. For multiple group comparisons, one-way ANOVA followed by Newman-Keul's multiple comparison test was performed. For two groups under two independent conditions, two-way ANOVA followed by Sidak's multiple comparison test was performed. All statistical analyses were done by GraphPad Prism. $\mathrm{P}>=0.05$ marked as ,ns' (not significant), $\mathrm{P}<0.05$ marked as “*'. Data are represented as mean \pm SD.

\section{Declarations}

\section{Ethics approval}

According to German law, no ethics approval is needed for fly experiments

\section{Consent for publication}

All authors read and approved the final manuscript.

\section{Availability of data}

All raw data and materials are available on request

\section{Competing interests}

The authors declare they have no competing interests.

\section{Funding}

This studied was funded by the Deutsche Forschungsgemeinschaft ME1922/15-1 and 17-1 to Axel Methner.

\section{Author contributions}

Conceptualization: LZ, AV, AM. Acquisition of data: LZ, SB (circadian clock experiment: VP). Analysis: LZ, SB, AM. Writing: LZ, AM.

\section{Acknowledgment}

We thank Teresa Alonso (University of Valladolid-CSIC, Spain) for donating the UAS-erGAP3 fly strain and Olaf Vef (University of Mainz, Germany) for the Mef2-Gal4 fly line. This work contains part of the doctoral thesis of Sebastian Buhr.

\section{References}

1. Gamboa-Tuz, S. D. et al. New insights into the phylogeny of the TMBIM superfamily across the tree of life: Comparative genomics and synteny networks reveal independent evolution of the $\mathrm{BI}$ and LFG families in plants. Mol. Phylogenet. Evol. 126, 266-278 (2018). 
2. Lisak, D. A. et al. The transmembrane Bax inhibitor motif (TMBIM) containing protein family: Tissue expression, intracellular localization and effects on the ER $\mathrm{CA}^{2+}$-filling state. Biochim Biophys Acta 1853, 2104-2114 (2015).

3. Zhao, H. et al. RECS1 deficiency in mice induces susceptibility to cystic medial degeneration. Genes Genet. Syst. 81, 41-50 (2006).

4. Zhao, G.-N. et al. Tmbim1 is a multivesicular body regulator that protects against nonalcoholic fatty liver disease in mice and monkeys by targeting the lysosomal degradation of TIr4. Nat Med 48, 467 (2017).

5. A new inhibitor of apoptosis from vaccinia virus and eukaryotes. 3, e17 (2007).

6. Oka, T. et al. Identification of a novel protein MICS1 that is involved in maintenance of mitochondrial morphology and apoptotic release of cytochrome c. Mol Biol Cell 19, 2597-2608 (2008).

7. Xu, Q. \& Reed, J. C. Bax inhibitor-1, a mammalian apoptosis suppressor identified by functional screening in yeast. Mol Cell 1, 337-346 (1998).

8. Bultynck, G. et al. The $\mathrm{C}$ terminus of Bax inhibitor-1 forms a Ca2+-permeable channel pore. Journal of Biological Chemistry 287, 2544-2557 (2012).

9. Chae, $\mathrm{H}$.-J. et al. $\mathrm{BI}-1$ regulates an apoptosis pathway linked to endoplasmic reticulum stress. Mol Cell 15, 355-366 (2004).

10. Westphalen, B. C., Wessig, J., Leypoldt, F., Arnold, S. \& Methner, A. BI-1 protects cells from oxygen glucose deprivation by reducing the calcium content of the endoplasmic reticulum. Cell Death Differ. 12, 304-306 (2005).

11. Bultynck, G., Kiviluoto, S. \& Methner, A. Bax inhibitor-1 is likely a pH-sensitive calcium leak channel, not a $\mathrm{H}+/ \mathrm{Ca}+$ exchanger. Science signaling 7, pe22 (2014).

12. Kiviluoto, $\mathrm{S}$. et al. Bax Inhibitor-1-mediated $\mathrm{Ca}(2+)$ leak is decreased by cytosolic acidosis. Cell Calcium 54, 186-192 (2013).

13. Chang, Y. et al. Structural basis for a $\mathrm{pH}$-sensitive calcium leak across membranes. Science 344, 1131-1135 (2014).

14. Kumar, K. N., Tilakaratne, N., Johnson, P. S., Allen, A. E. \& Michaelis, E. K. Cloning of cDNA for the glutamate-binding subunit of an NMDA receptor complex. Nature 354, 70-73 (1991).

15. Rojas-Rivera, D. et al. TMBIM3/GRINA is a novel unfolded protein response (UPR) target gene that controls apoptosis through the modulation of ER calcium homeostasis. Cell Death Differ. 19, 1013-1026 (2012).

16. Carrara, G. et al. Golgi anti-apoptotic proteins are highly conserved ion channels that affect apoptosis and cell migration. Journal of Biological Chemistry 290, 11785-11801 (2015).

17. A shear stress responsive gene product PP1201 protects against Fas-mediated apoptosis by reducing Fas expression on the cell surface. 16, 162-173 (2011).

18. Somia, N. V. et al. LFG: an anti-apoptotic gene that provides protection from Fasmediated cell death. Proc Natl Acad Sci USA 96, 12667-12672 (1999).

19. Hurtado de Mendoza, T. et al. Antiapoptotic protein Lifeguard is required for survival and maintenance of Purkinje and granular cells. Proc Natl Acad Sci USA 108, 1718917194 (2011).

20. Seitaj, B. et al. Transmembrane BAX Inhibitor-1 Motif Containing Protein 5 (TMBIM5) Sustains Mitochondrial Structure, Shape, and Function by Impacting the Mitochondrial Protein Synthesis Machinery. Cells 9, (2020).

21. Lisbona, F. et al. BAX inhibitor-1 is a negative regulator of the ER stress sensor IRE1alpha. Mol Cell 33, 679-691 (2009).

22. Up regulated Tmbim1 activation promotes high fat diet (HFD)-induced cardiomyopathy by enhancement of inflammation and oxidative stress. Biochem. Biophys. Res. Commun. 504, 797-804 (2018).

23. Deng, K.-Q. et al. Targeting Transmembrane BAX Inhibitor Motif Containing 1 Alleviates Pathological Cardiac Hypertrophy. Circulation 137, 1486-1504 (2018).

24. Komnig, D., Schulz, J. B., Reich, A. \& Falkenburger, B. H. Mice lacking Faim2 show increased cell death in the MPTP mouse model of Parkinson disease. J. Neurochem. 139, 848-857 (2016). 
25. Lisak, D. et al. BAX inhibitor-1 is a $\mathrm{Ca}(2+)$ channel critically important for immune cell function and survival. Cell Death Differ. 23, 358-368 (2016).

26. Philippaert, K. et al. Bax inhibitor-1 deficiency leads to obesity by increasing Ca2+dependent insulin secretion. J. Mol. Med. (2020). doi:10.1007/s00109-020-01914-x

27. Brand, A. H. \& Perrimon, N. Targeted gene expression as a means of altering cell fates and generating dominant phenotypes. Development 118, 401-415 (1993).

28. Blanchard, F. J. et al. The transcription factor Mef2 is required for normal circadian behavior in Drosophila. J Neurosci 30, 5855-5865 (2010).

29. Navas-Navarro, P. et al. GFP-Aequorin Protein Sensor for Ex Vivo and In Vivo Imaging of $\mathrm{Ca}(2+)$ Dynamics in High-Ca(2+) Organelles. Cell Chemical Biology 23, 738-745 (2016).

30. Renn, S. C., Park, J. H., Rosbash, M., Hall, J. C. \& Taghert, P. H. A pdf neuropeptide gene mutation and ablation of PDF neurons each cause severe abnormalities of behavioral circadian rhythms in Drosophila. Cell 99, 791-802 (1999).

31. Manning, L. et al. A hormonal cue promotes timely follicle cell migration by modulating transcription profiles. Mech. Dev. 148, 56-68 (2017).

32. Yoshida, H., Matsui, T., Yamamoto, A., Okada, T. \& Mori, K. XBP1 mRNA is induced by ATF6 and spliced by IRE1 in response to ER stress to produce a highly active transcription factor. Cell 107, 881-891 (2001).

33. Sone, M., Zeng, X., Larese, J. \& Ryoo, H. D. A modified UPR stress sensing system reveals a novel tissue distribution of IRE1/XBP1 activity during normal Drosophila development. Cell Stress Chaperones 18, 307-319 (2013).

34. Dietzl, G. et al. A genome-wide transgenic RNAi library for conditional gene inactivation in Drosophila. Nature 448, 151-156 (2007).

35. Ni, J.-Q. et al. A genome-scale shRNA resource for transgenic RNAi in Drosophila. Nat Methods 8, 405-407 (2011).

36. Carrara, G., Saraiva, N., Gubser, C., Johnson, B. F. \& Smith, G. L. Sixtransmembrane topology for Golgi anti-apoptotic protein (GAAP) and Bax inhibitor 1 (BI-1) provides model for the transmembrane Bax inhibitor-containing motif (TMBIM) family. Journal of Biological Chemistry 287, 15896-15905 (2012).

37. Frakes, A. E. et al. Four glial cells regulate ER stress resistance and longevity via neuropeptide signaling in C. elegans. Science 367, 436-440 (2020).

38. Henikoff, S. \& Henikoff, J. G. Amino acid substitution matrices from protein blocks. Proc Natl Acad Sci USA 89, 10915-10919 (1992).

39. Simossis, V. A., Kleinjung, J. \& Heringa, J. Homology-extended sequence alignment. Nucleic Acids Res. 33, 816-824 (2005).

40. Dereeper, A. et al. Phylogeny.fr: robust phylogenetic analysis for the non-specialist. Nucleic Acids Res. 36, W465-9 (2008).

41. Edgar, R. C. MUSCLE: multiple sequence alignment with high accuracy and high throughput. Nucleic Acids Res. 32, 1792-1797 (2004). 


\section{Figure Legends}

Figure 1: Phylogenetic relationship of fly and human TMBIM proteins

Phylogenetic analysis of the sequence alignment of human and Drosophila TMBIM proteins identifies CG2076 and CG1287 as orthologues for TMBIM5 and CG7188 as TMBIM6, TMBIM4 is most probably CG30370 while the relationship of TMBIM1-3 to their fly orthologues is less clear. The bacterial homologue BsYetJ is most closely related to TMBIM5 and TMBIM6. The branch support values are indicated above the branches. The phylogenetic tree was created using the Maximum Likelihood algorithm with the BLOSUM62 substitution matrix and 100 bootstrap trials. The dashed lines indicate closest homologues according to BLASTP.

Figure 2 Expression levels and phenotype of CG7188 (dmTMBIM6) knockdown in flies

a) Temporal and tissue expression of CG7188/dmTMBIM5 from Flybase (http://flybase.org/).

b) Viability of knockdown (KD) with different GAL4 drivers and the knockdown efficiency of two UAS-CG7188-RNAi lines (\#1, \#2) assessed by quantitative PCR in whole larvae of Mef2>>CG7188-RNAi. c) Effects of neural KD by elav-GAL4 of CG7188 on eclosing, wing phenotype and life span. d) Crawling ability of larvae of muscular KD (Mef2>>CG7188-RNAi). e) Activity of Pdf>>CG7188-RNA under 12/12 hours light/dark cycle. Data are represented as mean \pm SD. One-way ANOVA followed by Newman-Keul's multiple comparison test for multiple comparison and un-paired t-test for two groups comparison were performed using GraphPad Prism. * $p<0.05$.

Figure 3 Expression levels and phenotype of CG2076 (dmTMBIM5) knockdown in flies

a) Temporal and tissue expression of CG2076/dmTMBIM5 from Flybase (http://flybase.org/).

b) Viability of CG2076 knockdown (KD) flies by different GAL4 drivers and the KD efficiency of UAS-CG2076-RNAi (\#1, \#2) assessed by quantitative PCR in whole larvae of Mef2>>CG2076-RNAi. c) Eclosing rate of pupa and lifespan of elav>>CG2076-RNAi. d) Crawling ability of larvae, climbing ability and lifespan of adults of Mef2>>CG2076-RNAi. e) Activity of Pdf $\gg>C G 2076-R N A i$ flies under 12/12 hours light/dark cycle. Data are represented as mean \pm SD. One-way ANOVA followed by Newman-Keul's multiple comparison test for multiple comparison and two-way ANOVA followed by Sidak's multiple comparison test for multiple comparison under two independent conditions were performed using GraphPad Prism. * $p<0.05$.

Figure 4 Expression levels and phenotype of CG30379 (putative dmTMBIM4) knockdown in flies

a) Temporal and tissue expression of CG30379/dmTMBIM4 from Flybase (http://flybase.org/).

b) Viability of CG30379 knockdown (KD) flies by different GAL4 drivers and the KD efficiency of UAS-CG30379-RNAi (\#1, \#2) assessed by quantitative PCR in fly heads of 
elav>>CG30379-RNAi (\#1) and elav>>CG30379-RNAi (\#2). c) Eclosing rate of pupa and lifespan of elav>>CG30379-RNAi. d) Crawling ability of larvae, climbing ability and lifespan of adults of Mef2>>CG30379-RNAi. e) Activity of Pdf >>CG30379-RNAi flies under 12/12 hours light/dark cycle. Data are represented as mean \pm SD. One-way ANOVA followed by NewmanKeul's multiple comparison test for multiple comparison and two-way ANOVA followed by Sidak's multiple comparison test for multiple comparison under two independent conditions were performed using GraphPad Prism. ${ }^{*} p<0.05$.

Figure 5 Expression levels and phenotype of CG3798 (putative dmTMBIM3) knockdown in flies

a) Temporal and tissue expression of CG3798/dmTMBIM3 from Flybase (http://flybase.org/).

b) Viability of CG3798 knockdown (KD) flies by different GAL4 drivers and the KD efficiency of UAS-CG3798-RNAi (\#1, \#2) assessed by quantitative PCR in whole adults of tub>>CG3798-RNAi. c) Eclosing rate of pupa and lifespan of elav>>CG3798-RNAi. d) Crawling ability of larvae, climbing ability and lifespan of adults of Mef2>>CG3798-RNAi. e) Activity of Pdf $>>C G 3798-R N A i$ flies under 12/12 hours light/dark cycle. Data are represented as mean $\pm S D$. One-way ANOVA followed by Newman-Keul's multiple comparison test for multiple comparison and two-way ANOVA followed by Sidak's multiple comparison test for multiple comparison under two independent conditions were performed using GraphPad Prism. ${ }^{*} \mathrm{p}<0.05$.

Figure 6 Expression levels and phenotype of CG3814 (putative dmTMBIM2) knockdown in flies

a) Temporal and tissue expression of CG3814/dmTMBIM2 from Flybase (http://flybase.org/).

b) Viability of CG3814 knockdown (KD) flies by different GAL4 drivers and the knockdown efficiency of UAS- CG3814-RNAi (\#1, \#2) assessed by quantitative PCR in whole adults of tub>>CG3814-RNAi. c) Eclosing rate of pupa and lifespan of elav>>CG3814-RNAi. d) Crawling ability of larvae, climbing ability and lifespan of adults of Mef2>>CG3814-RNAi. e) Activity of Pdf $>>C G 3814-R N A i$ flies under 12/12 hours light/dark cycle. Data are represented as mean \pm SD. One-way ANOVA followed by Newman-Keul's multiple comparison test for multiple comparison and two-way ANOVA followed by Sidak's multiple comparison test for multiple comparison under two independent conditions were performed using GraphPad Prism. ${ }^{*} p<0.05$.

Figure 7 Expression levels and phenotype of CG9722 (putative dmTMBIM1) knockdown in flies

a) Temporal and tissue expression of CG9722/dmTMBIM1 from Flybase (http://flybase.org/).

b) Viability of CG9722 knockdown (KD) flies by different GAL4 drivers and the KD efficiency of UAS- CG9722-RNAi (\#1) assessed by quantitative PCR in whole larvae of Mef2>>CG9722- 
RNAi. c) Hatching rate of pupa, adults' wing phenotype and lifespan of elav>>CG9722-RNAi. d) Crawling ability of larvae, lifespan of a few surviving adults of Mef2>>CG9722-RNAi. e) Activity of Pdf $>>$ CG9722-RNAi flies under 12/12 hours light/dark cycle. f)g) The transcriptional level of CG7188 and CG9722 in Mef>>CG9722-RNAi and Mef>>CG7188-RNAi respectively. Data are represented as mean \pm SD. One-way ANOVA followed by Newman-Keul's multiple comparison test for multiple comparison and unpaired t-test for two groups comparison were performed using GraphPad Prism. * $\mathrm{p}<0.05$.

Figure 8 ER calcium and ER stress in TMBIM KD flies and summary of the phenotypes

a) Exemplary confocal images of ER calcium (erGAP3) in Pdf-GAL4 driven TMBIM knockdown (KD) flies and b) Quantification. Scale bar, $5 \mu \mathrm{m}$. c) Relative location of primers/probe used to detect spliced and unspliced Xbp1 and d) Quantification of ER stress using the ratio of spliced and unspliced Xbp1 in heads from elav-GAL4 driven TMBIM KD flies. Each data point represents one animal in b) and one group (10 fly heads per group) in d). Data are represented as mean \pm SD. One-way ANOVA followed by Newman-Keul's multiple comparison test was performed using GraphPad Prism. * $p<0.05$. e) Summary of phenotypes observed in this study.

Supplemental Figure S1 Expression levels of CG9722 (dmTMBIM1) in the CG9722 RNAi\#2 line and MiMIC line

a) tub>>CG9722-RNAi\#2 (BL6679) showed increased transcriptional levels of CG9722 assessed by quantitative qPCR in whole adults. b) CG9722-MiMIC line (BL44979), which is supposed to be a CG9722 knockout does not alter transcriptional levels of CG9722. c) Location of primers in respect to the CG9722 MiMIC insertion cassette. In homozygous CG9722 MiMIC flies, PCR using primer pair 1 amplifies a product of over 500 bp indicating no insertion of the MiMIC cassette. Primer pair 2 suggests the existence of the MiMIC cassette in the genome. Primer pairs $1^{\text {for }} / 2^{\text {rev }}$ and $2^{\text {for }} / 1^{\text {rev }}$ respectively $1^{\text {for }} / 2^{\text {for }}$ and $1^{\text {rev }} / 2^{\text {rev }}$ did not result in any PCR product in line with the lack of the MIMIC cassette within the CG9722 locus. Data are represented as mean \pm SD. Unpaired t-test were performed using GraphPad Prism. ${ }^{*} p<0.05$.

Supplemental Table S1 RNAi lines used in this study. 
Figure 1 Phylogenetic relationship of TMBIM proteins in humans and flies

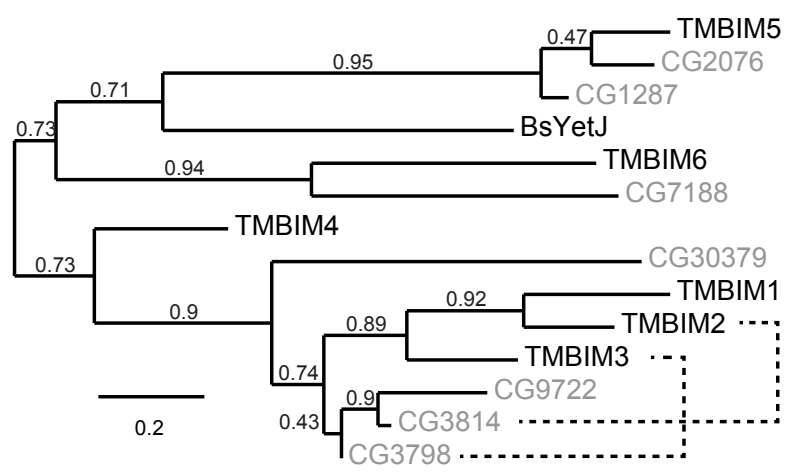


Figure 2

a CG7188/dmTMBIM6 Tissue expression level 131 central nervous system, larvae L3 head, virgin 1-day female
head, virgin 4-day female head, virgin 20-day female head, mated 1-day female head, mated 20-day female head, mated 1-day male head, mated 4-day male head, mated 20-day male salivary gland, white prepupae digestive system, larvae $L 3$ wandering digestive system, 1-day adult digestive system, 4-day adult digestive system, 20-day adult fat body, larvae L3 wandering fat body, white prepupae fat body, pupae P8 carcass, larvae L3 wandering carcass, 1-day adult carcass, 4-day adult carcass, 20-day adult ovary, virgin 4-day female ovary, mated 4-day female testis, mated 4-day male accessory gland, mated 4-day male

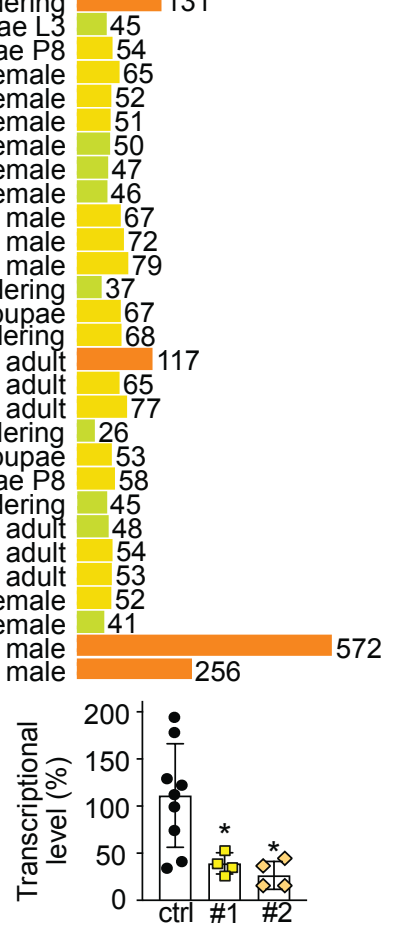

b

\begin{tabular}{cccc}
\hline CG7188 & \multicolumn{3}{c}{ Viability } \\
\cline { 2 - 4 } RNAi line & tub & elav & Mef2 \\
\hline \#1 & lethal & viable & lethal \\
\#2 & lethal & viable & lethal
\end{tabular}

imaginal disc, larvae L3 wandering salivary gland, larvae L3 wandering $37^{79}$
C elav-Gal4
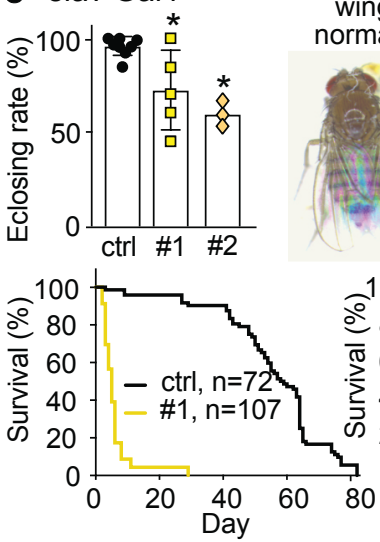

e Pdf-Gal4

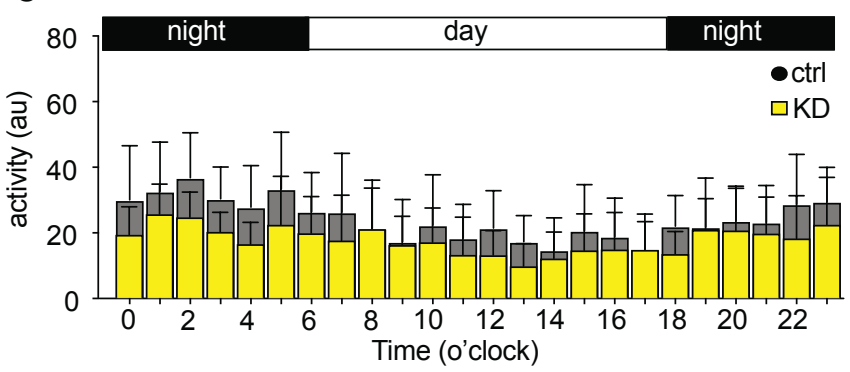

wing phenotype normal uninflated
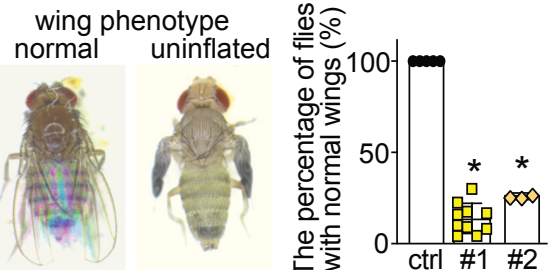

d Mef2-Gal4

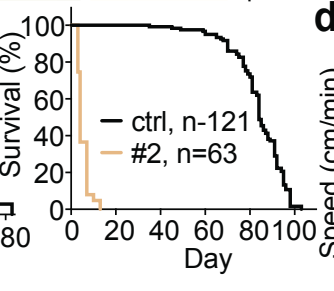

की

ctrl

KD 
Figure 3

a CG2076/dmTMBIM5 Tissue imaginal disc, larvae L3 wandering central nervous system, pupae $\mathrm{P} 8$ head, virgin 1-day female head, virgin 4-day female head, mated 1-day female head, mated 4-day female head, mated 20-day female head, mated 1-day male head, mated 4-day male head, mated 20 -day male salivary gland, larvae L 3 wandering salivary gland, white prepupae digestive system larvae $L 3$ wandering digestive system, 1-day adult digestive system, 4-day adult digestive system, 4 -day adult fat body, fad, larvae $L 3$ wandering fat body, white prepupae at body, pupae P8 carcass, larvae L3 wandering carcass, 1-day adul carcass, 4-day adult carcass, 20-day adult ovary, virgin 4-day female ovary, mated 4-day female testis, mated 4-day male accessory gland, mated 4-day male

b

\begin{tabular}{|c|c|c|c|}
\hline \multirow{2}{*}{$\begin{array}{l}\text { CG2076 } \\
\text { RNAi line }\end{array}$} & \multicolumn{3}{|c|}{ Viability } \\
\hline & tub & elav & Mef2 \\
\hline \#1 & lethal & viable & viable \\
\hline \#2 & lethal & viable & viable \\
\hline
\end{tabular}

expression level

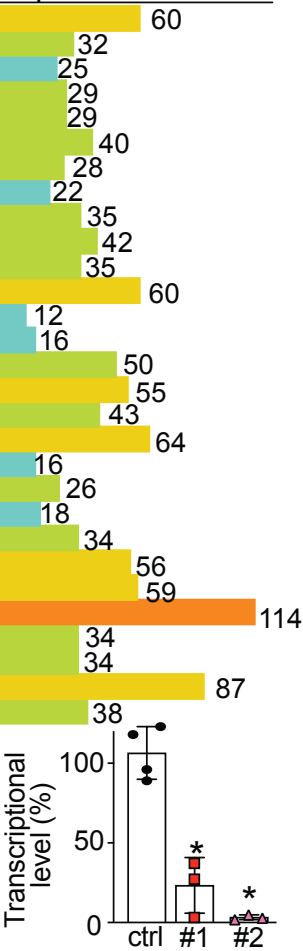

C elav-Gal4
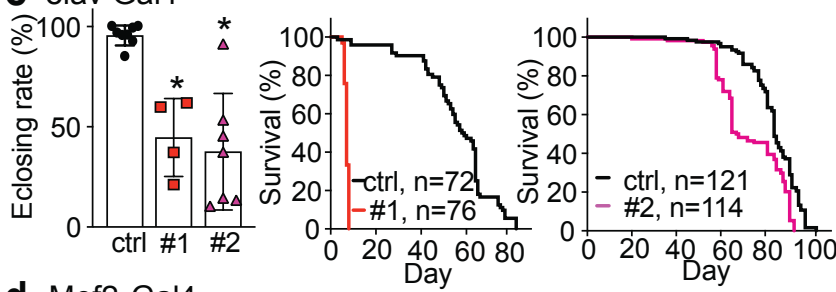

d Mef2-Gal4
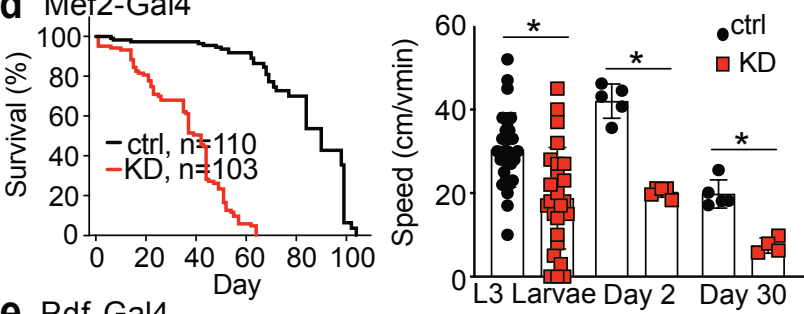

e Pdf-Gal4

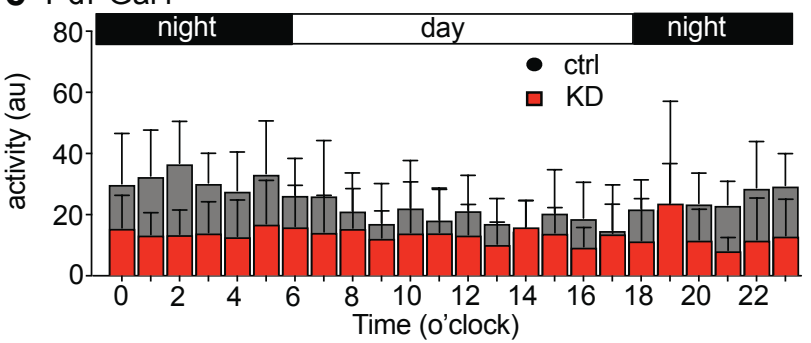


Figure 4

acG30379/dmTMBIM4

Tissue

expression level

imaginal disc, larvae L3 wandering

central nervous system, larvae L3

central nervous system, pupae P8

head, virgin 1-day female

head, virgin 4-day female

head, virgin 20-day female

head, mated 1-day female

head, mated 4-day female

head, mated 20-day female

head, mated 1-day male

head mated 4-day male

head mated 20-day male

salivary gland, larvae L 3 wandering

salivary gland, white prepupae

salivary gland, white prepupae

digestive system, 1-day adult

digestive system, 4-day adult

digestive system, 20-day adult

fat body, larvae L 3 wandering

fat body, white prepupae

fat white prepupae

carcass, larvae L3 wandering

carcass, 1-day adult

carcass, 4-day adult

carcass, 20-day adult

ovary, virgin 4-day female

ovary, mated 4-day female

testis, mated 4-day male

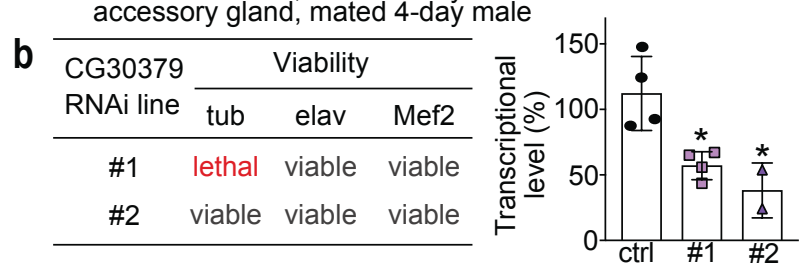

C elav-Gal4

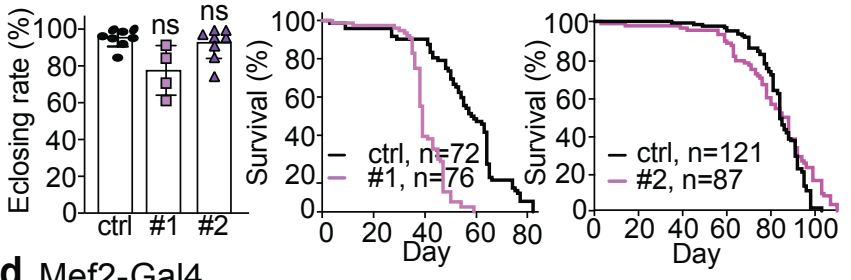

d Mef2-Gal4
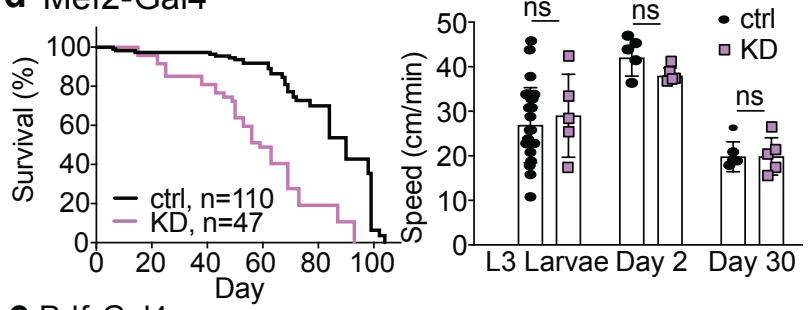

e Pdf-Gal4

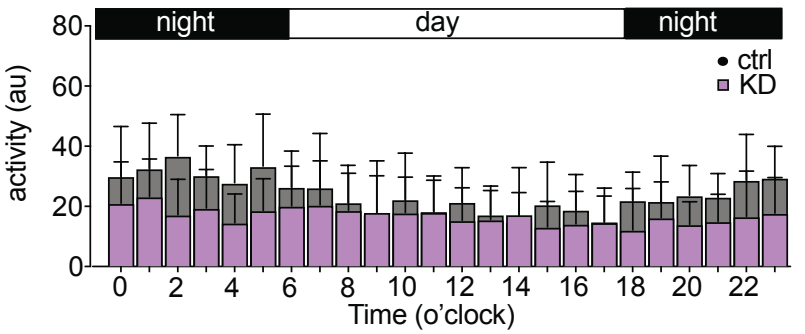


Figure 5

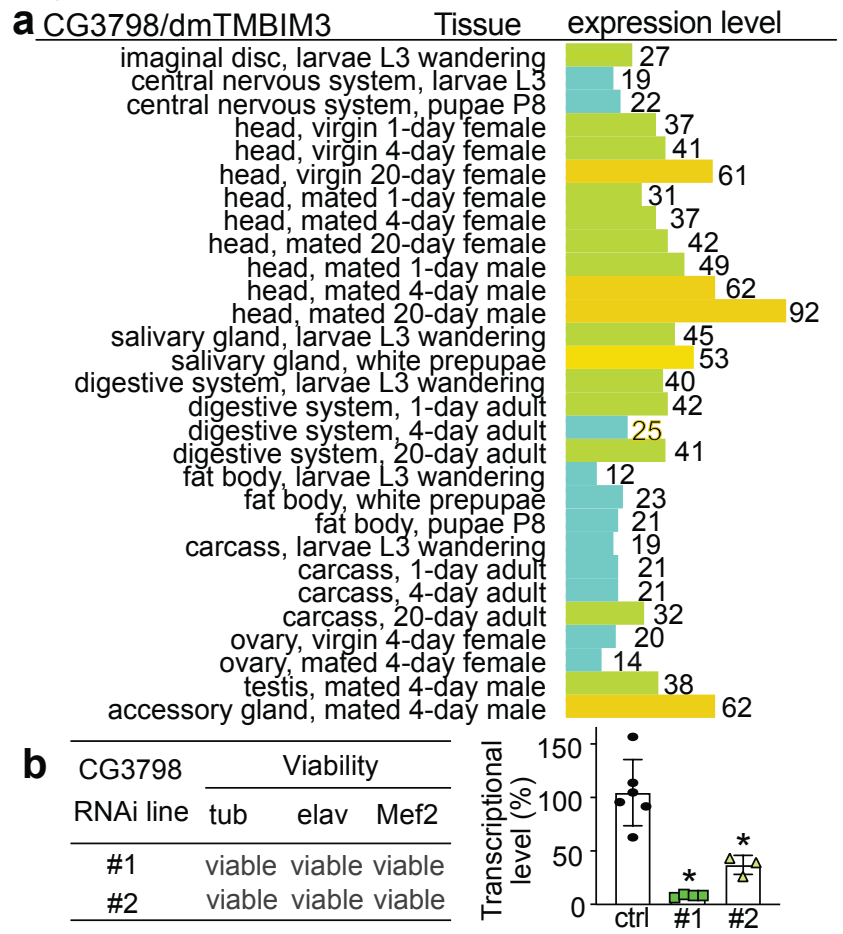

C elav-Gal4

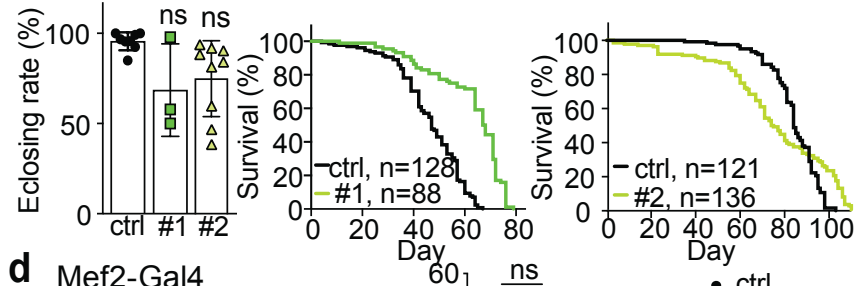

\section{d Mef2-Gal4} Day

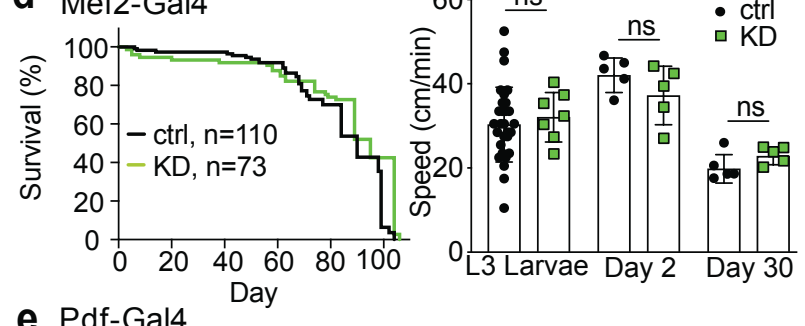

e Pdf-Gal4

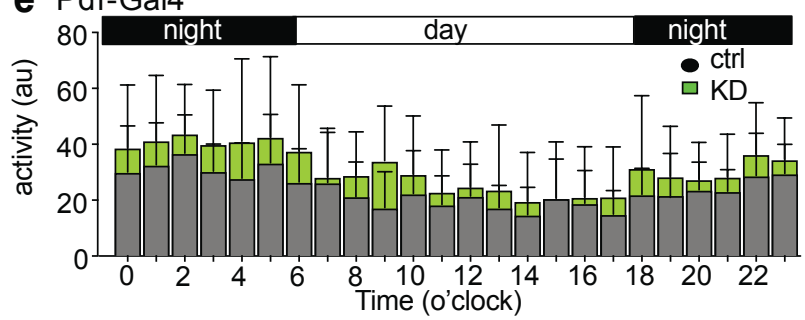


Figure 6

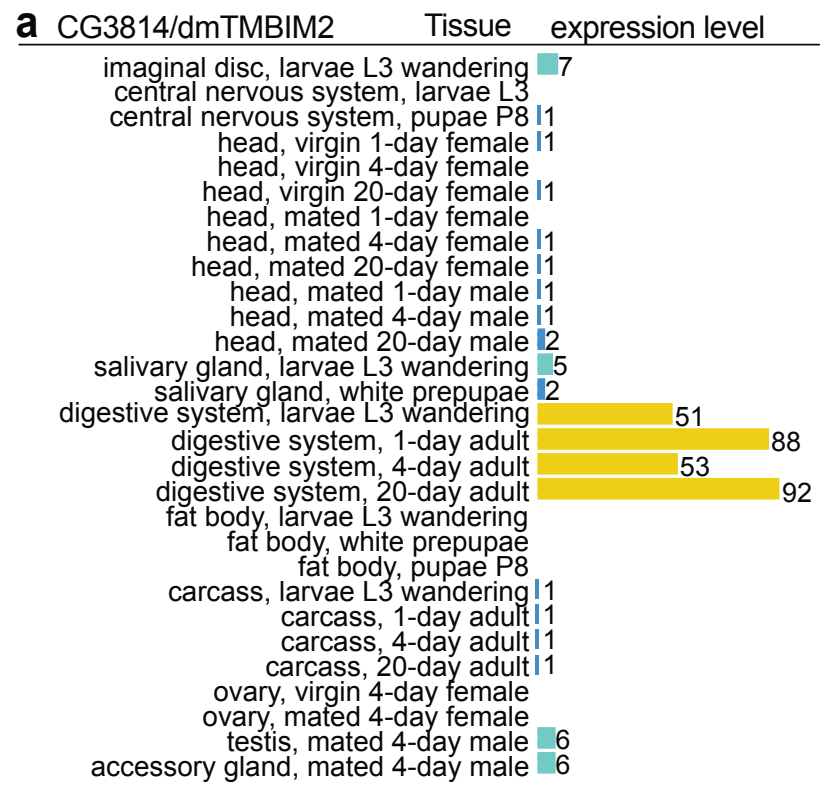

\begin{tabular}{|c|c|c|c|}
\hline \multirow{2}{*}{$\begin{array}{l}\text { CG3814 } \\
\text { RNAi line }\end{array}$} & \multicolumn{3}{|c|}{ Viability } \\
\hline & Tub & elav & mef2 \\
\hline \#1 & viable & viable & viable \\
\hline \#2 & viable & viable & viable \\
\hline
\end{tabular}

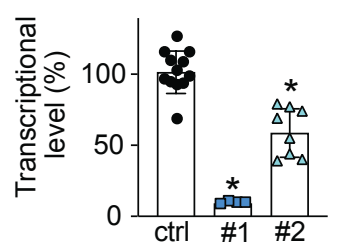

C elav-Gal4
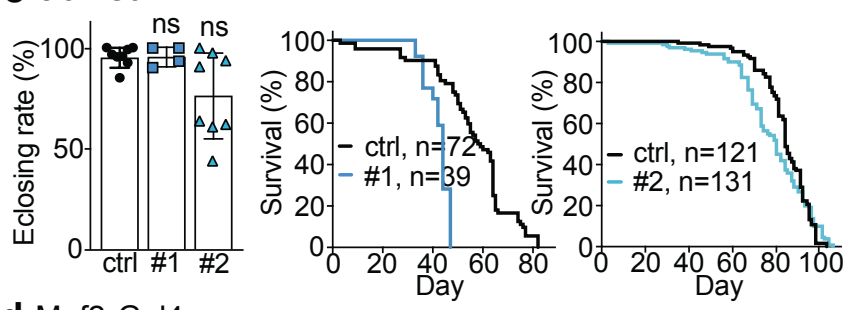

d Mef2-Gal4
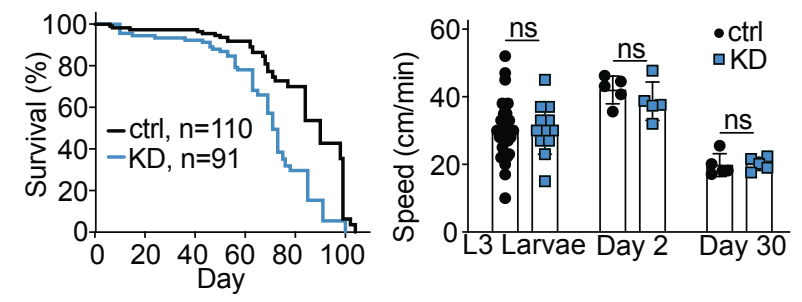

e Pdf-Gal4

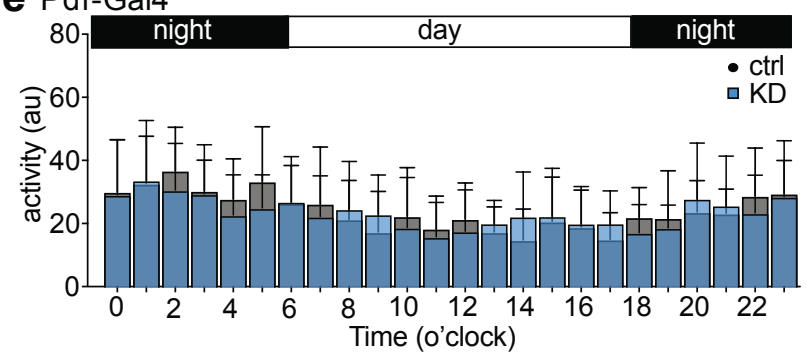


Figure 7

a CG9722/dmTMBIM1 Tissue Expression level imaginal disc, larvae L3 wandering 12 central nervous system, larvae central nervous system, pupae head, virgin 1-day female head, virgin 4-day female head, virgin 2 -day emale head, mated 4-day ema head, mated 20-day female head, mated 1-day male head, mated 4-day male head, mated 20 -day male salivary gland, larvae 3 wandering digestive system larvae 13 wandering

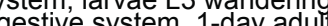
igestive system, 4 -day adu digestive system, 4 -day adul fat body larvae 13 wandering fat body, white prepupae 12

carcass, larvae 3 , pupae carcass, 1-day adul carcass, 4-day adult carcass, 20-day adult ovary, virgin 4-day temale ovary, mated 4-day female accessory gland, mated 4-day male

b

\begin{tabular}{|c|c|c|c|}
\hline CG9722 & & iability & \\
\hline RNAi line & tub & elav & Mef2 \\
\hline \#1 & lethal & viable & lethal \\
\hline
\end{tabular}

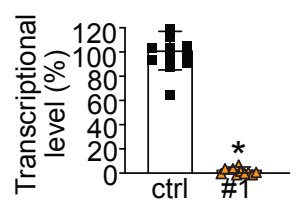

\section{C elav-Gal4}

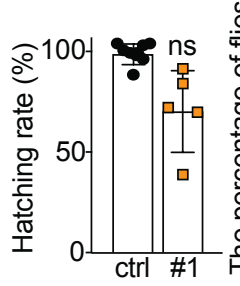

d Mef2-Gal4
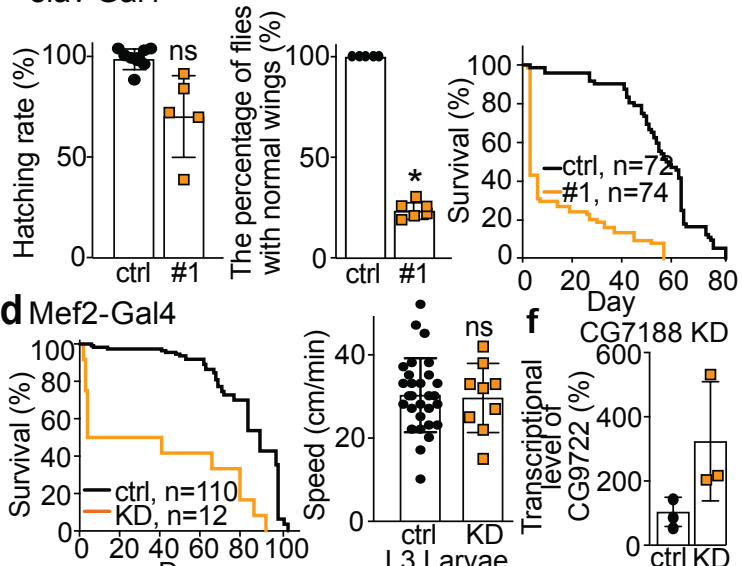

$$
\text { - } f \text { Day }
$$

- ns f CG7188 KD

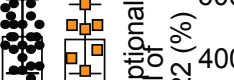

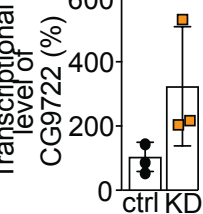

e Pdf-Gal4

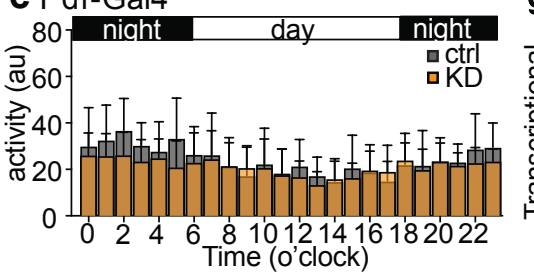

g CG9722 KD

1000 $\overline{\sqrt{0}} \bigcirc 800$ 要 
Figure 8

a $\bullet$ ctrl

-ctrl 7188

口CG7188

口CG30379

-CG3798

-CG3814

ctrl .
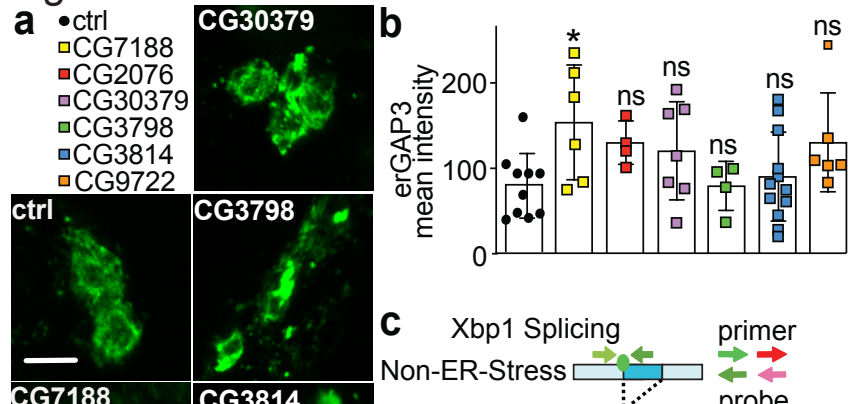

C Xbp1 Splicing primer Non-ER-Stress $\square$

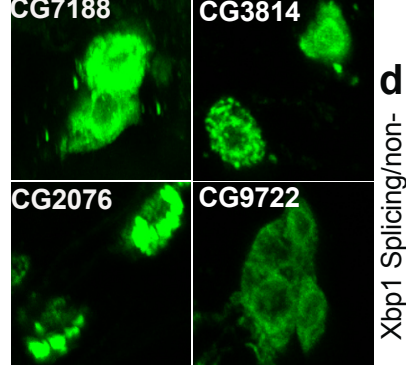
ER-Stress probe

d

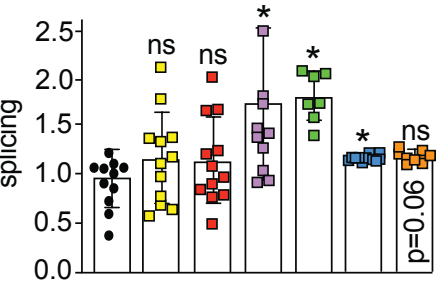

\begin{tabular}{|l|l|l|l|l|l|l|}
\hline $\mathbf{e}$ & CG7188 & CG2076 & CG30379 & CG3798 & CG3814 & CG9722 \\
\hline
\end{tabular}

\begin{tabular}{|r|c|c|c|c|c|c|}
\hline \multicolumn{7}{|c|}{ Ubiquitous knockdown (tub-Gal4) } \\
\hline viability & lethal & lethal & lethal & viable & viable & lethal \\
\hline \multicolumn{7}{|c|}{ pan-neuronal knockdown (elav-Gal4) } \\
\hline viability & viable & viable & viable & viable & viable & viable \\
hatching & low & low & normal & normal & normal & normal \\
lifespan & short & short & short & long & short & short \\
Wing & abnor. & normal & normal & normal & normal & abnor. \\
ER stress & normal & normal & high & high & high & normal
\end{tabular}

muscule-specific knockdown (mef2-Gal4)

\begin{tabular}{|r|c|c|c|c|c|c|}
\hline \multicolumn{7}{|c|}{ muscule-specific knockdown (mef2-Gal4) } \\
\hline viability & lethal & viable & viable & viable & viable & semi-lethal \\
Crawling & normal & slow & normal & normal & normal & normal \\
lifespan & $X$ & short & short & normal & short & short \\
Climbing & $X$ & slow & normal & normal & normal & X \\
\hline \multicolumn{7}{|c|}{ circadian clock neuron cluster knockdown(pdf-Gal4) } \\
\hline activity & hypo & hypo & hypo & hyper & normal & hypo \\
ER calcium & high & normal & normal & normal & normal & normal \\
\hline
\end{tabular}




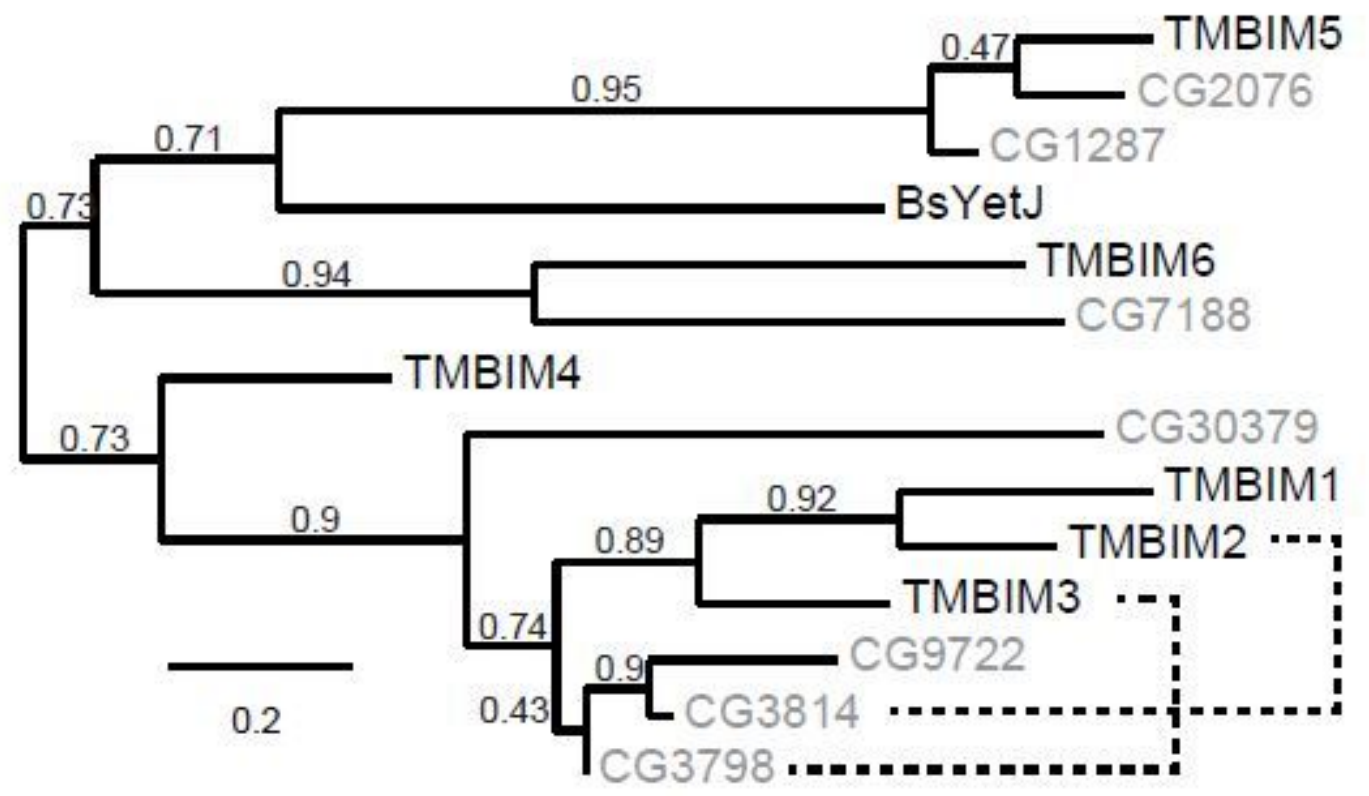

Figure 1

Phylogenetic relationship of fly and human TMBIM proteins Phylogenetic analysis of the sequence alignment of human and Drosophila TMBIM proteins identifies CG2076 and CG1287 as orthologues for TMBIM5 and CG7188 as TMBIM6, TMBIM4 is most probably CG30370 while the relationship of TMBIM13 to their fly orthologues is less clear. The bacterial homologue BsYetJ is most closely related to TMBIM5 and TMBIM6. The branch support values are indicated above the branches. The phylogenetic tree was created using the Maximum Likelihood algorithm with the BLOSUM62 substitution matrix and 100 bootstrap trials. The dashed lines indicate closest homologues according to BLASTP. 
a cG7188/dmTMBIM6 Tissue

imaginal disc, larvae L3 wandering

central nervous system, larvae L3
central nervous system, pupae P8

head, virgin 1-day female

head, virgin 4-day female

head virgin 20-day female

head, mated 1-day female

head, mated 4-day female

head, mated 20-day female

head mated 1-day male

head' mated 4-day male

head mated 20-day male

salivary gland, larvae L3 wandering salivary gland, white prepupae digestive system, larvae L3 wandering

digestive system, 1-day adult

digestive system, 4-day adult digestive system, 4-day adult
digestive system, 20-day adult

fat body, larvae L3 wandering

fat body, white prepupae fat body, pupae P8

carcass, larvae L 3 wandering carcass, 1-day adult carcass, 4-day adult carcass, 20-day adult

ovary, virgin 4-day female

ovary mafed 4-day female

testis, mated 4-day male

accessory gland, mated 4-day male

expression level

b

\begin{tabular}{|c|c|c|c|}
\hline G7188 & \multicolumn{3}{|c|}{ Viability } \\
\hline RNAi li & tub & elav & Mef2 \\
\hline \#1 & lethal & viable & lethal \\
\hline \#2 & lethal & viable & lethal \\
\hline
\end{tabular}

45131
54

65

51

50

47

67

72
79

67

68

117

77

53

53

45

48

54

52

41

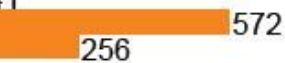

$256 \quad 572$

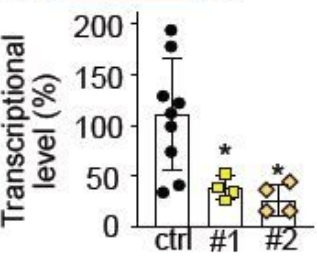

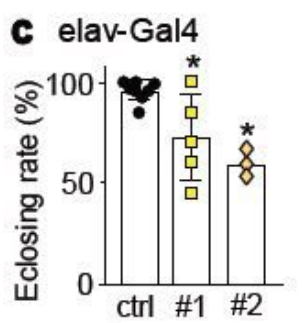

wing phenotype

normal uninflated
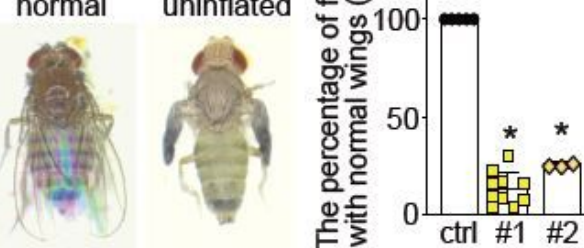

d Mef2-Gal4
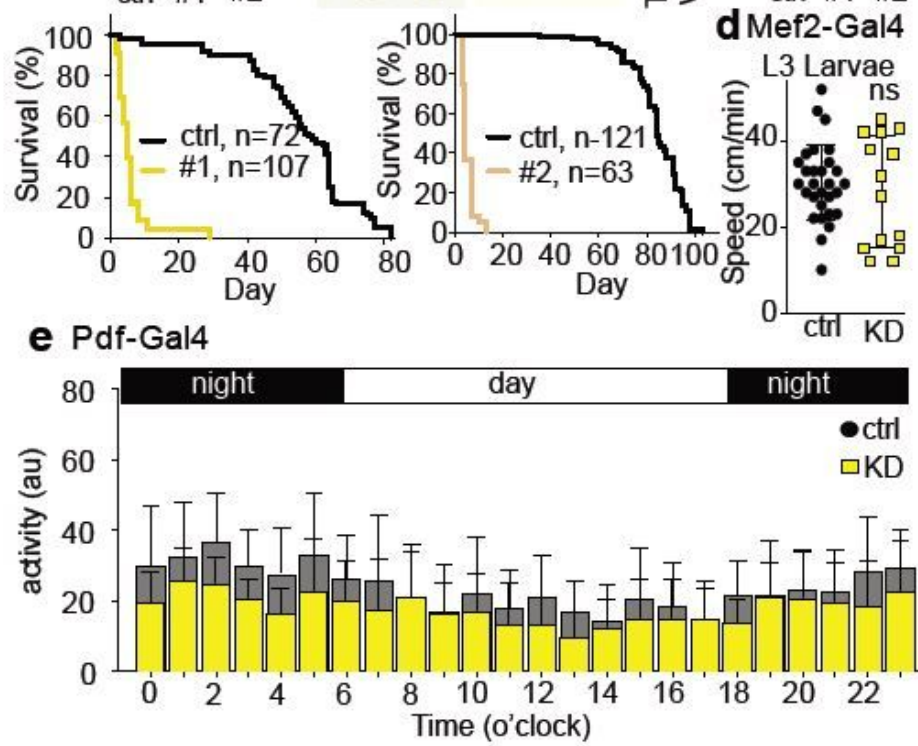

Figure 2

Expression levels and phenotype of CG7188 (dmTMBIM6) knockdown in flies a) Temporal and tissue expression of CG7188/dmTMBIM5 from Flybase (http://flybase.org/). b) Viability of knockdown (KD) with different GAL4 drivers and the knockdown efficiency of two UAS-CG7188-RNAi lines (\#1, \#2) assessed by quantitative PCR in whole larvae of Mef2>>CG7188-RNAi. c) Effects of neural KD by elavGAL4 of CG7188 on eclosing, wing phenotype and life span. d) Crawling ability of larvae of muscular KD (Mef2>>CG7188-RNAi). e) Activity of Pdf >>CG7188-RNA under 12/12 hours light/dark cycle. Data are represented as mean \pm SD. One-way ANOVA followed by Newman-Keul's multiple comparison test for multiple comparison and un-paired t-test for two groups comparison were performed using GraphPad Prism. ${ }^{*} \mathrm{p}<0.05$. 
acG2076/dmTMBIM5 Tissue

imaginal disc, larvae L3 wandering

central nervous system, larvae L3

central nervous system, pupae P8

head, virgin 1-day female

head, virgin 4-day female head virgin 20-day female

head, mated 1-day female

head, mated 4-day female

head, mated 20-day female head, mated 1-day male head, mated 4-day male head mated 20-day male

salivary gland, larvae L 3 wandering salivary gland, white prepupae digestive system, larvae L3 wandering digestive system, 1-day adult digestive system 4-day adult digestive system, 20-day adult fat body, larvae $L 3$ wandering fat body, white prepupae fat body pupae P8

carcass, larvae L3 wandering carcass, 1-day adult carcass, 4-day adult carcass, 20-day adult ovary, virgin 4-day female ovary, mated 4-day female

testis, mated 4-day male accessory gland, mated 4-day male

b

\begin{tabular}{cccc}
\hline \multirow{2}{*}{ RG2076 } & \multicolumn{3}{c}{ Viability } \\
\cline { 2 - 3 } RNAi line & tub & elav & Mef2 \\
\hline \multirow{2}{*}{$\# 1$} & lethal & viable & viable \\
$\# 2$ & lethal & viable & viable \\
\hline
\end{tabular}

expression leve 60

25

29

40

22

35

42

35

12
16

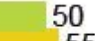

$43^{55}$

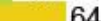

16

$18^{26}$

34

56

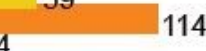

34
34 87

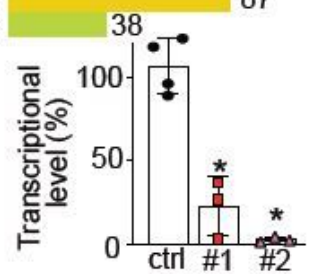

C elav-Gal4
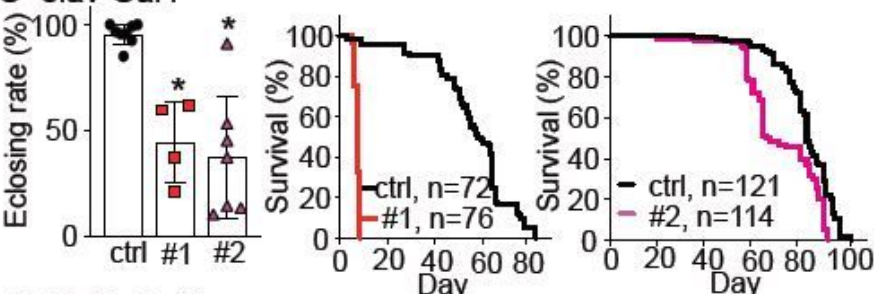

d Mef2-Gal4
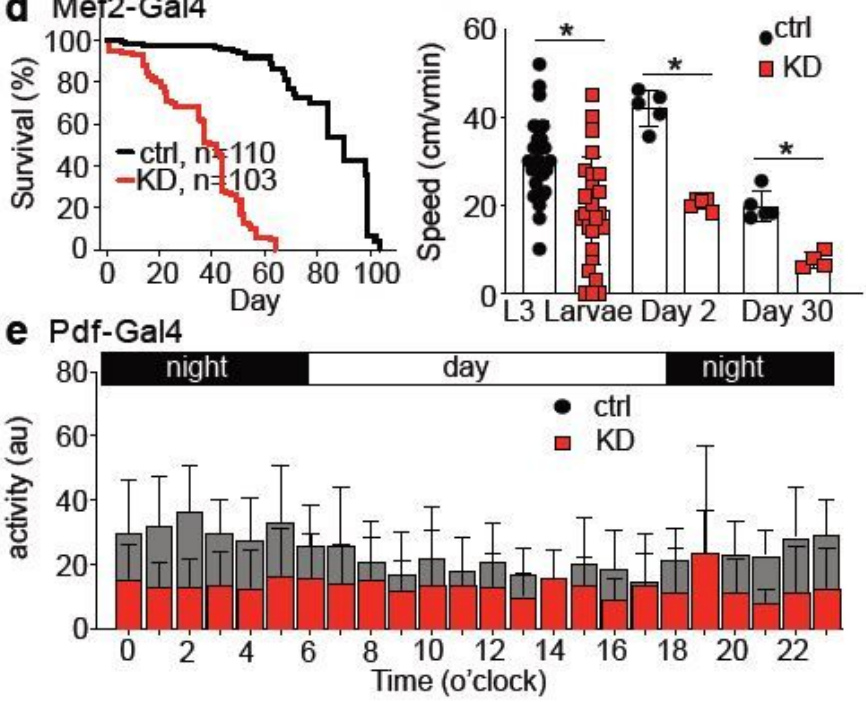

Figure 3

Expression levels and phenotype of CG2076 (dmTMBIM5) knockdown in flies a) Temporal and tissue expression of CG2076/dmTMBIM5 from Flybase (http://flybase.org/). b) Viability of CG2076 knockdown (KD) flies by different GAL4 drivers and the KD efficiency of UAS-CG2076-RNAi (\#1, \#2) assessed by quantitative PCR in whole larvae of Mef2>>CG2076-RNAi. c) Eclosing rate of pupa and lifespan of elav >>CG2076-RNAi. d) Crawling ability of larvae, climbing ability and lifespan of adults of Mef2>>CG2076-RNAi. e) Activity of Pdf >>CG2076-RNAi flies under 12/12 hours light/dark cycle. Data are represented as mean \pm SD. One-way ANOVA followed by Newman-Keul's multiple comparison test for multiple comparison and two-way ANOVA followed by Sidak's multiple comparison test for multiple comparison under two independent conditions were performed using GraphPad Prism. * ${ }^{\star}<0.05$. 
acG30379/dmTMBIM4 Tissue expression level imaginal disc, larvae L3 wandering central nervous system, larvae L 3

central nervous system, pupae P8 3 head, virgin 1-day female head, virgin 4-day female head virgin 20-day female

head, mated 1-day female

head mated 4 day female

head, mated 20-day female head, mated 1-day male head, mated 4-day male head mated 20-day male

salivary gland, larvae L 3 wandering

salivary gland, white prepupae digestive system, larvae $\mathrm{L} 3$ wandering

digestive system, 1-day adul

digestive system, 4-day adul

digestive system 20-day adult

fat body larvae 13 wandering

fat body white prepupa

carcass, larvae L 3 wandering

carcass, 1-day adul

carcass 4-day adu

carcass, 20-day adult

ovary, virgin 4-day female

ovary mated 4-day female

testis, mated 4-day male

\begin{tabular}{|c|c|c|c|}
\hline \multirow{2}{*}{$\begin{array}{l}\text { CG30379 } \\
\text { RNAi line }\end{array}$} & \multicolumn{3}{|c|}{ Viability } \\
\hline & tub & elav & Mef2 \\
\hline$\# 1$ & lethal & viable & viable \\
\hline \#2 & viable & viable & viable \\
\hline
\end{tabular}

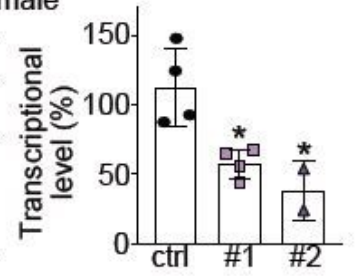

c elav-Gal4
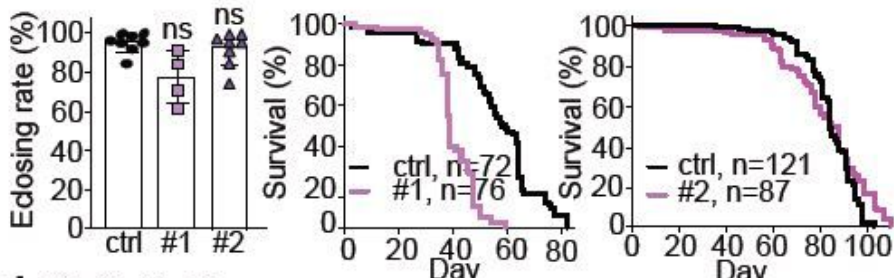

d Mef2-Gal4

Day

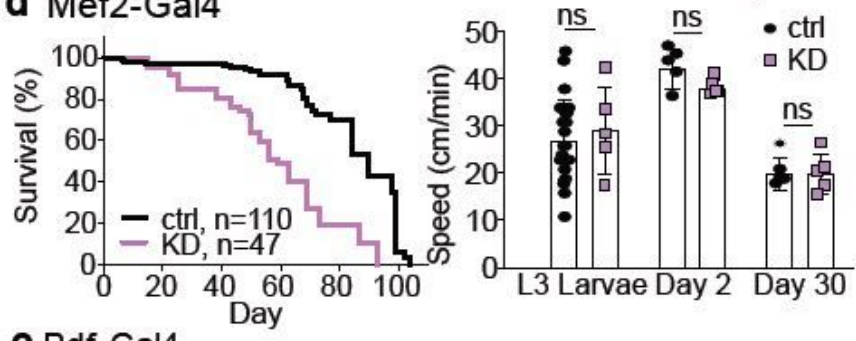

e Pdf-Gal4

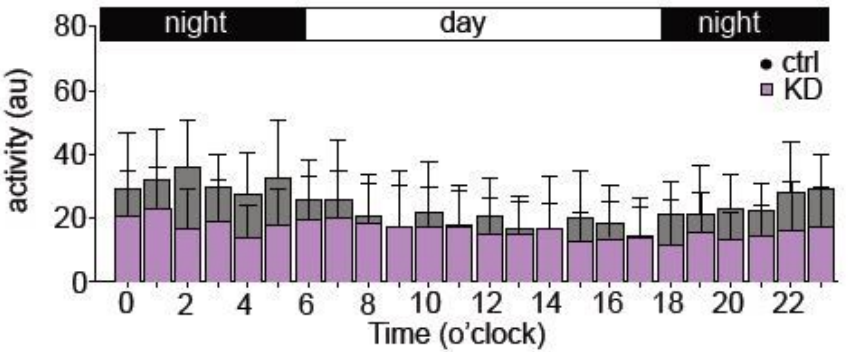

Figure 4

Expression levels and phenotype of CG30379 (putative dmTMBIM4) knockdown in flies a) Temporal and tissue expression of CG30379/dmTMBIM4 from Flybase (http://flybase.org/). b) Viability of CG30379 knockdown (KD) flies by different GAL4 drivers and the KD efficiency of UAS-CG30379-RNAi (\#1, \#2) assessed by quantitative PCR in fly heads of 17 elav>>CG30379-RNAi (\#1) and elav>>CG30379-RNAi (\#2). c) Eclosing rate of pupa and lifespan of elav>>CG30379-RNAi. d) Crawling ability of larvae, climbing ability and lifespan of adults of Mef2 >>CG30379-RNAi. e) Activity of Pdf >>CG30379-RNAi flies under $12 / 12$ hours light/dark cycle. Data are represented as mean \pm SD. One-way ANOVA followed by NewmanKeul's multiple comparison test for multiple comparison and two-way ANOVA followed by Sidak's multiple comparison test for multiple comparison under two independent conditions were performed using GraphPad Prism. * $\mathrm{p}<0.05$. 
a CG3798/dmTMBIM3

Tissue expression level

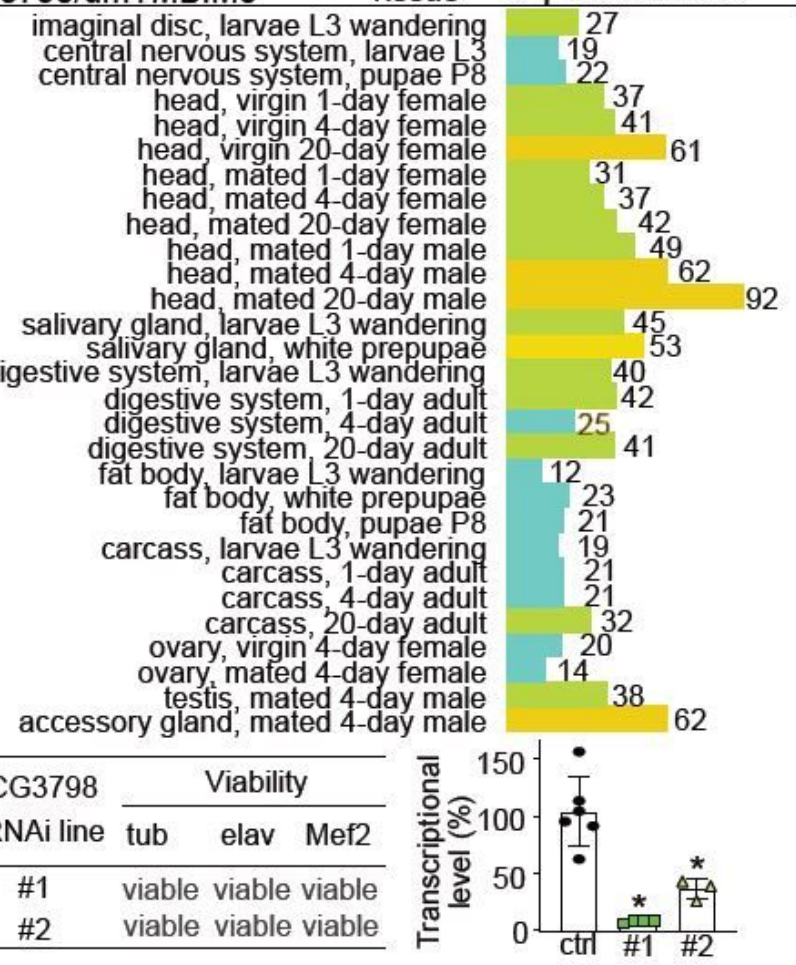

C elav-Gal4
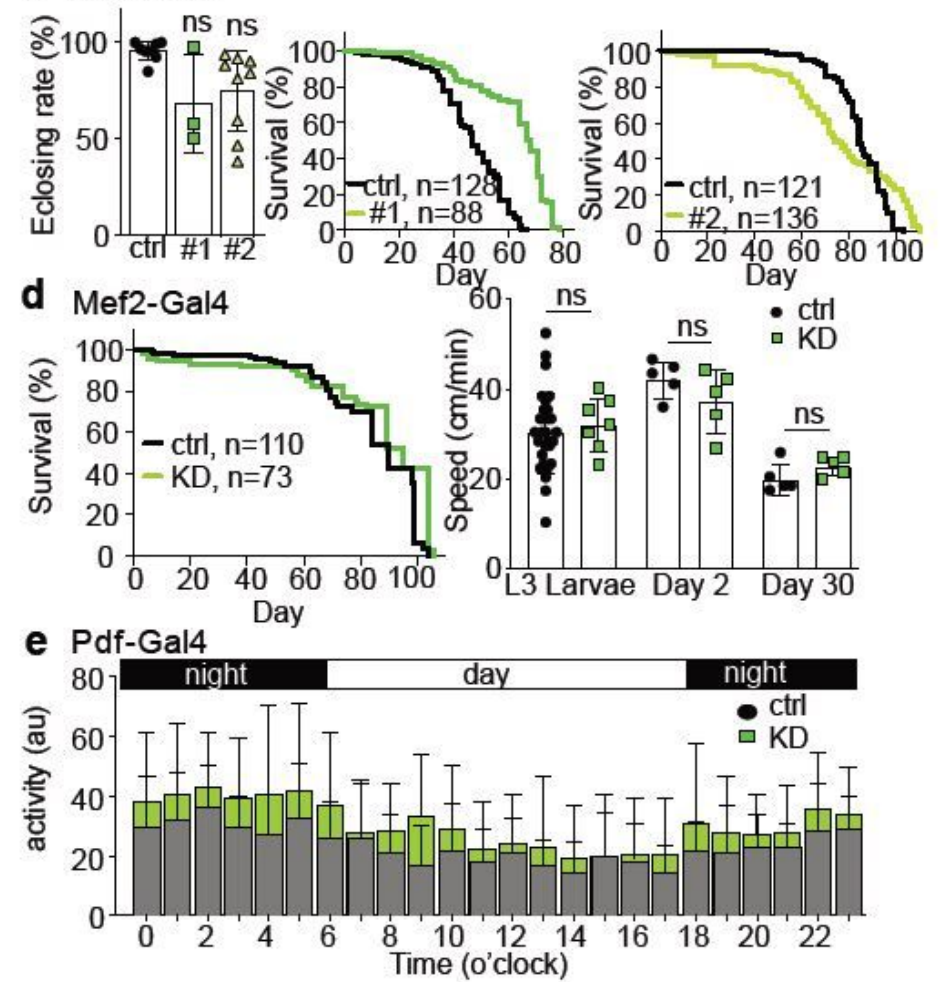

Figure 5

Expression levels and phenotype of CG3798 (putative dmTMBIM3) knockdown in flies a) Temporal and tissue expression of CG3798/dmTMBIM3 from Flybase (http://flybase.org/). b) Viability of CG3798 knockdown (KD) flies by different GAL4 drivers and the KD efficiency of UAS-CG3798-RNAi (\#1, \#2) assessed by quantitative PCR in whole adults of tub>>CG3798-RNAi. c) Eclosing rate of pupa and lifespan of elav >>CG3798-RNAi. d) Crawling ability of larvae, climbing ability and lifespan of adults of Mef2 >>CG3798-RNAi. e) Activity of Pdf >>CG3798-RNAi flies under 12/12 hours light/dark cycle. Data are represented as mean \pm SD. One-way ANOVA followed by Newman-Keul's multiple comparison test for multiple comparison and two-way ANOVA followed by Sidak's multiple comparison test for multiple comparison under two independent conditions were performed using GraphPad Prism. ${ }^{*} p<0.05$. 

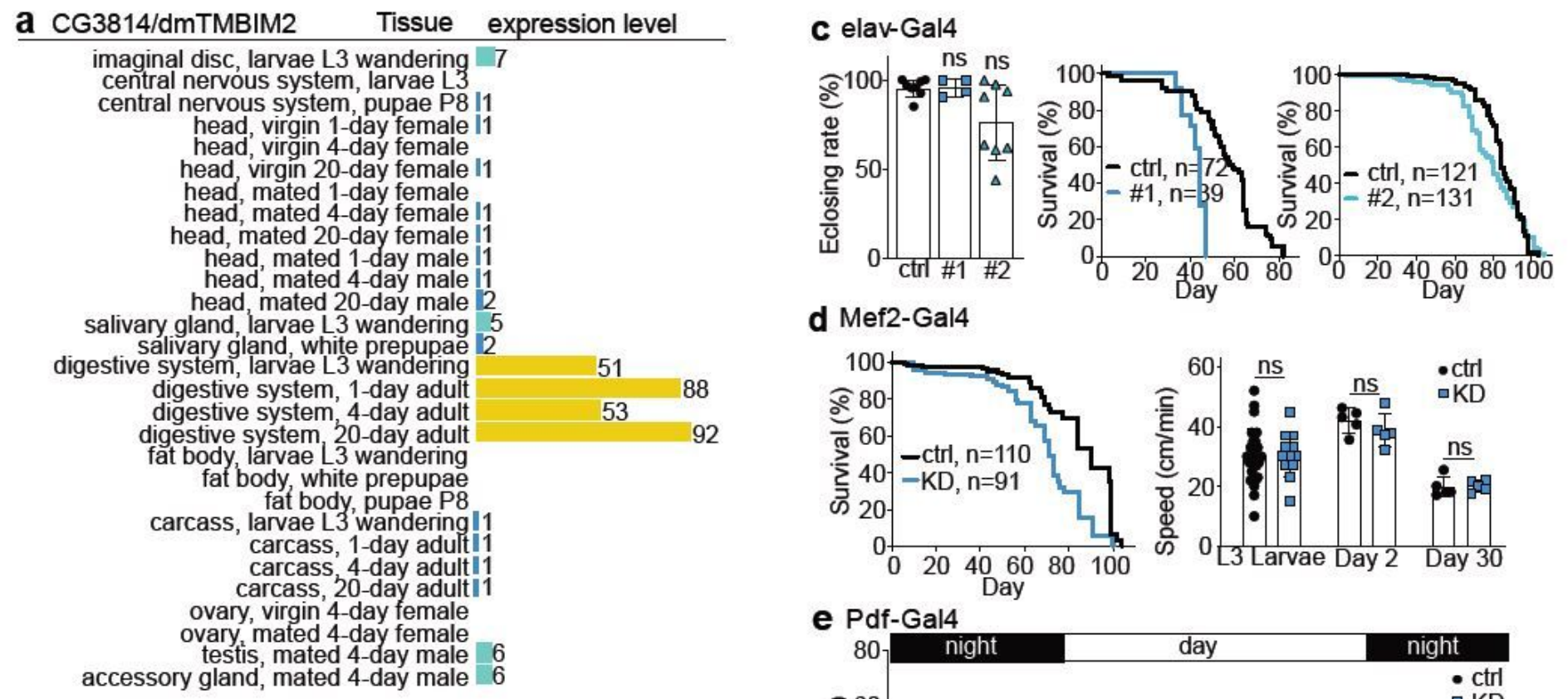

\begin{tabular}{|c|c|c|c|}
\hline \multirow{2}{*}{$\begin{array}{l}\text { CG3814 } \\
\text { RNAi line }\end{array}$} & \multicolumn{3}{|c|}{ Viability } \\
\hline & Tub & elav & mef2 \\
\hline$\# 1$ & viable & viable & viable \\
\hline$\# 2$ & viable & viable & viable \\
\hline
\end{tabular}

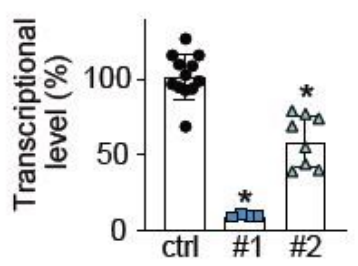

\section{d Mef2-Gal4}
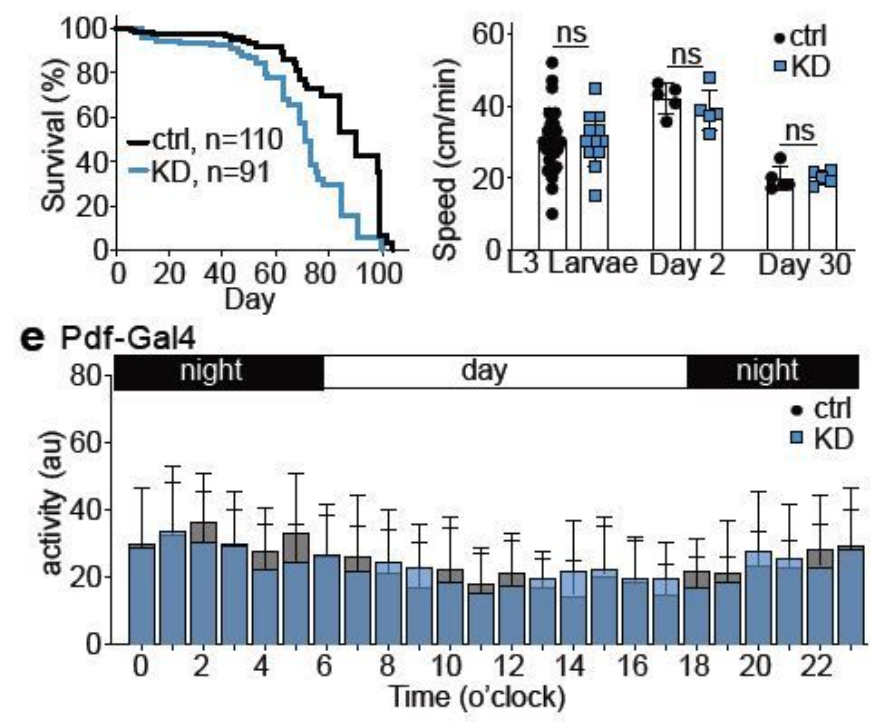

\section{Figure 6}

Expression levels and phenotype of CG3814 (putative dmTMBIM2) knockdown in flies a) Temporal and tissue expression of CG3814/dmTMBIM2 from Flybase (http://flybase.org/). b) Viability of CG3814 knockdown (KD) flies by different GAL4 drivers and the knockdown efficiency of UAS- CG3814-RNAi (\#1, \#2) assessed by quantitative PCR in whole adults of tub>>CG3814-RNAi. c) Eclosing rate of pupa and lifespan of elav>>CG3814-RNAi. d) Crawling ability of larvae, climbing ability and lifespan of adults of Mef2>>CG3814-RNAi. e) Activity of Pdf >>CG3814-RNAi flies under 12/12 hours light/dark cycle. Data are represented as mean \pm SD. One-way ANOVA followed by Newman-Keul's multiple comparison test for multiple comparison and two-way ANOVA followed by Sidak's multiple comparison test for multiple comparison under two independent conditions were performed using GraphPad Prism. ${ }^{*} \mathrm{p}<0.05$. 
a cG9722/dmTMBIM1 Tissue Expression level

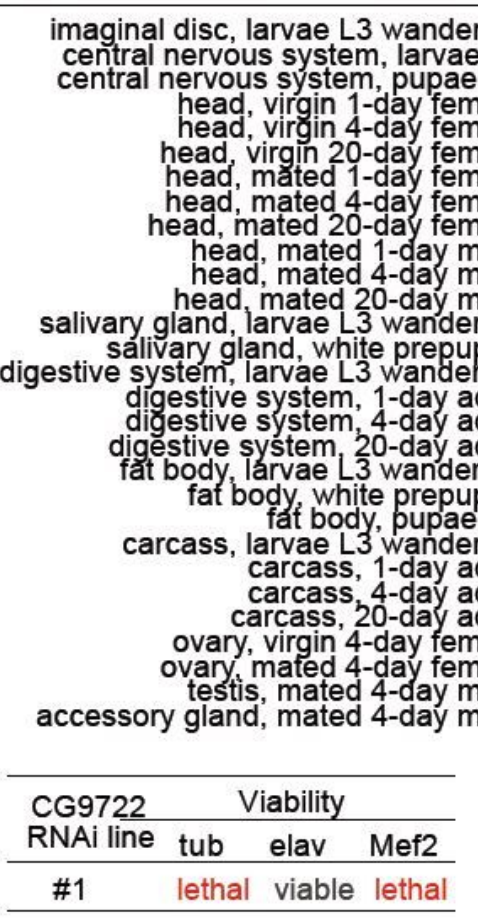

60
C elav-Gal4
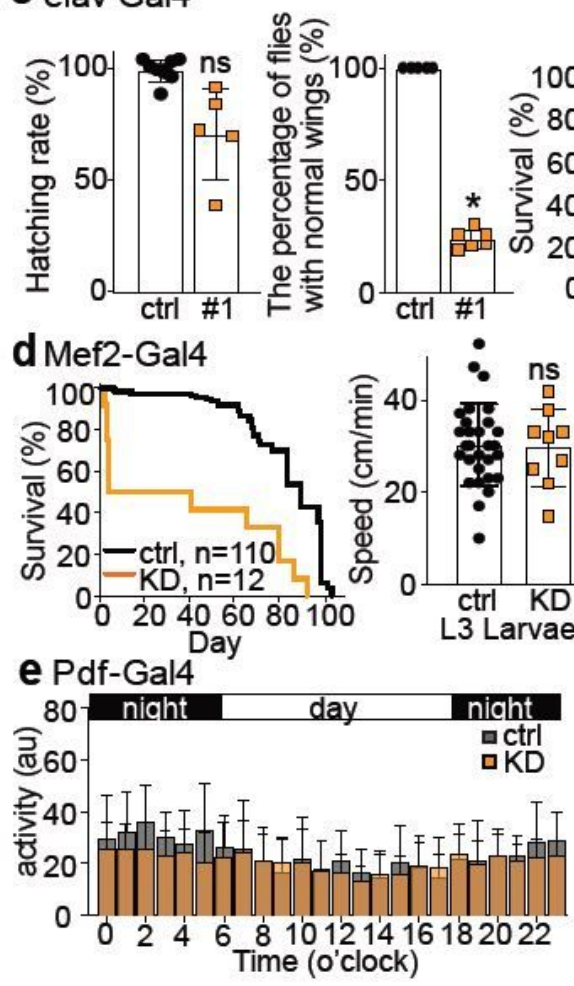

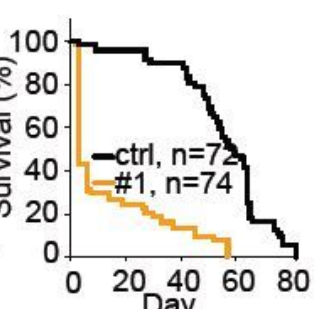

Day

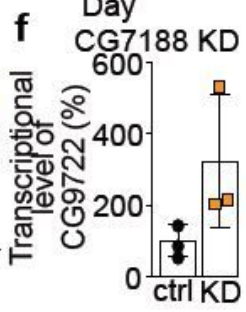

g CG9722 KD

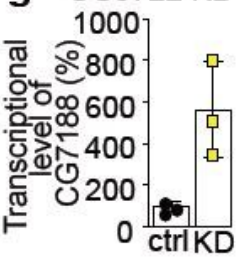

\section{Figure 7}

Expression levels and phenotype of CG9722 (putative dmTMBIM1) knockdown in flies a) Temporal and tissue expression of CG9722/dmTMBIM1 from Flybase (http://flybase.org/). b) Viability of CG9722 knockdown (KD) flies by different GAL4 drivers and the KD efficiency of UAS- CG9722-RNAi (\#1) assessed by quantitative PCR in whole larvae of Mef2>>CG9722- 18 RNAi. c) Hatching rate of pupa, adults' wing phenotype and lifespan of elav>>CG9722-RNAi. d) Crawling ability of larvae, lifespan of a few surviving adults of Mef2>>CG9722-RNAi. e) Activity of Pdf >>CG9722-RNAi flies under 12/12 hours light/dark cycle. f)g) The transcriptional level of CG7188 and CG9722 in Mef>>CG9722-RNAi and Mef $>>C G 7188-R N A i$ respectively. Data are represented as mean \pm SD. One-way ANOVA followed by Newman-Keul's multiple comparison test for multiple comparison and unpaired t-test for two groups comparison were performed using GraphPad Prism. ${ }^{*} p<0.05$. 


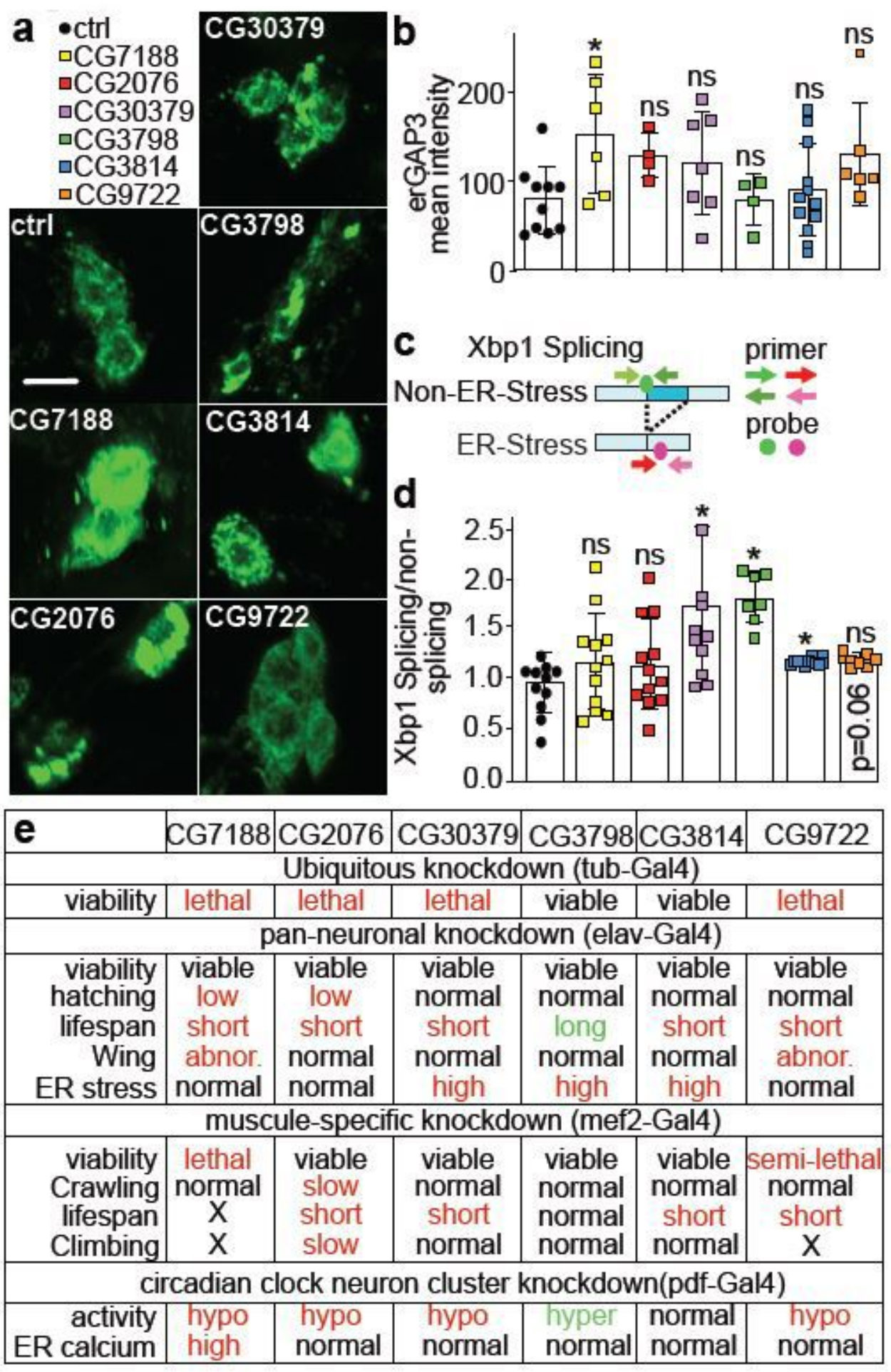

Figure 8

ER calcium and ER stress in TMBIM KD flies and summary of the phenotypes a) Exemplary confocal images of ER calcium (erGAP3) in Pdf-GAL4 driven TMBIM knockdown (KD) flies and b) Quantification. Scale bar, $5 \mu \mathrm{m}$. c) Relative location of primers/probe used to detect spliced and unspliced Xbp1 and d) Quantification of ER stress using the ratio of spliced and unspliced Xbp1 in heads from elav-GAL4 driven TMBIM KD flies. Each data point represents one animal in b) and one group (10 fly heads per group) in 
d). Data are represented as mean \pm SD. One-way ANOVA followed by Newman-Keul's multiple comparison test was performed using GraphPad Prism. ${ }^{\star} p<0.05$. e) Summary of phenotypes observed in this study.

\section{Supplementary Files}

This is a list of supplementary files associated with this preprint. Click to download.

- SupplementaryInformation.docx 